\title{
Long-wave Marangoni instability with vibration
}

\author{
By UWE THIELE, JOSÉ M. VEGA \\ AND EDGAR KNOBLOCH \\ Max-Planck-Institut für l'hysik komplexer Systeme, Nöthnitzer Str. 38, D-01187 Dresden, Germany \\ E.I.S.I. $\Lambda$ eronáuticos, Universidad Politécnica de Madrid, Plaza Cardenal Cisneros 3, \\ 28040 Madrid, Spain \\ Department of Physics, University of California, Berkeley, CA 94720, USA
}

\begin{abstract}
The effect of vertical vibration on the long-wave instability of a Marangoni system is studied. The vibration augments the stabilizing effect of surface tension in bounded systems. In laterally unbounded systems nonlinear terms can stabilize non-flat states and prevent the appcarance of dry spots. 'l'he effect of a slight inclination of the system is also considered.
\end{abstract}

\section{Introduction}

Thermocapillary phenomena (Scriven \& Sternling 1960; Davis 1987) are of interest in many applications, including matcrials processing and crystal growth in a microgravity environment (Jurish \& Löser 1990; Kuhlmann 1999), and coating (Cazabat et al. 1990; Kataoka \& Troian 1997) and drying ('Thess \& Boos 1999; Matar \& Craster 2001) processes under terrestrial conditions, and often serve as a source of both convection and instabilitics. Although Bcnard was alrcady aware of the rolc of surface tension gradients in his seminal experiment (Bénard 1900), the ability of thermocapillary stresses to drive convection was elucidated only by Rayleigh (1916) as part of his explanation of the appearance of convection cells in thin films (sec also Block 1956; Koschmieder 1993). In such films buoyancy is typically negligible and convection is driven by thermocapillary effects alone. The simplest configuration exhibiting this effect consists of a horizontal fluid layer supported by a hot plate and bounded above by a colder free surface. This system, known as the Marangoni Bénard system, has been used extensively to investigate both thermocapillary phenomena (Davis 1987; Koschmieder 1993; Bragard \& Velarde 1998) and the formation of patterns (Cross \& Hohenberg 1993). Some of the thermocapillary instabilities that are observed are long wave (VanI Iook et al. 1997) and have therefore also been studied in the context of thin films (see Oron, Davis \& Bankoff 1997 for a recent revicw, as well as Boos \& Thess 1999; Oron 2000; Bestchorn, Pototsky \& Thicle 2003; Thiele \& Knobloch 2004).

In this paper we examine the effect of vertical vibration on these phenomena. We focus on films that are sufficiently thin that buoyancy effects are small, or equivalently consider low-gravity environments, where residual acceleration, due to crew manocuvring and machinery, has a significant impact on both matcrial processing systems and on-board experiments (see Skarda 2001 for references). In general, residual oscillatory acceleration, or $g$-jitter, is broad-band and varies randomly both 
in magnitude and direction. This acceleration is usually transmitted through the support structure or container walls of the fluid system in question. Thus the actual excitation of the fluid is transmitted via the narrow-band structural response centred on the natural frequencies of the container or support structure. Although the random nature of the vibration and the presence of overtones cannot be ignored, the usual first step (which also provides physical insight into fundamental mechanisms) is to take the vibration as monochromatic, with a constant amplitude and direction.

As is well known, an inverted pendulum can be stabilized by vertical vibration of the support (Landau \& Lifshitz 1987). In fluid systems the same principle has becn used to stabilize the Rayleigh-Taylor instability in an upside-down container (Lapuerta, Mancebo \& Vega 2001 and references therein) and interfacial instabilities of films flowing down an inclined plane (Lin \& Chen 1998 and references therein). In particular, Wolf (1970) showed experimentally that normal vibration may suppress the Rayleigh Taylor instability of a flat film in the short-wavelength regime, i.e. in relatively thick films. Mechanical and thermal vibration has also been studied in connection with the Rayleigh-Bénard system (Gershuni, Zhukhovitskii \& Jurkov 1970; Gresho \& Sani 1970), directional solidification (Murray, Coriell \& McFadden 1991; Wheeler et al. 1991), and doubly diffusive convection (Gershuni et al. 1997 and references therein), and used to control both thermocapillary convection (Nicolas, Rivas \& Vega 1998 and references therein) and thermocapillary instabilitics. Although this approach is attractive a number of complications remain. For example, normal vibration cannot stabilize the conductive state in an unbounded film (Woods \& Lin 1995), while tangential vibration (Or 1997) is only effective for vibration frequencies that are not too large. In either case the stabilization results are obtained by solving a Floquet problem, and are in general quite subtle and non-intuitive. Morcover, the presence of lateral boundaries may be important. For example, bounded films can be stabilized by normal vibration, and the stabilizing eflect is greatest for large vibration frequencies. In addition, the vibration affects the convective states that result from any instability. Others (Or \& Kelly 2002) have used temperature modulation with similar ideas in mind, while Skarda (2001) considered vibration perpendicular to the supporting plate, but ignored free-surface deformation even though longwave instabilitics can only occur in the presence of frec-surface deformation when gravitational effects are small (Davis 1987). As shown by Or et al. (1999) the resulting Marangoni instability can be suppressed by an active fecdback control. We mention also that the influence of normal vibration on the shape of pendant droplets was investigated by (Faraday 1831, \$44 50), while Gavrilyuk, Lukovsky \& Timokha (2004) have used a variational principle to study this system in the large-frequency limit.

These considerations motivate the present study. We focus on the effect of normal vibration on the long-wave Marangoni instability of thin films on horizontal and inclined planes, and investigate its influence on the linear and nonlinear stability of uniform films. In particular, we describe the effects of vibration on both periodic wavetrains, and stationary and sliding drops.

The problem is formulated in $\$ 2$, followed in $\$ 3$ by a derivation of the relevant long-wave equation for a vibrated thin film under the assumption of no thermal expansion. This equation is analysed and integrated numerically in $\$ 4$ for a film on a horizontal support. Unbounded films on an inclined plane are considered in $\$ 5$. Concluding remarks follow in $\$ 6$. Possible effects of thermal expansion are discussed in an Appendix. 


\section{Formulation}

We consider a largc-aspect-ratio container of depth $d^{*}$ and cross-scction $\Omega^{*}$, vibrated vertically with an amplitude $a^{*}$ and a frequency $\omega^{*}$, where the superscript * denotes dimensional magnitudes. The bottom of the container is maintained at a fixed temperature $T_{0}^{*}$ and the system loses heat through the frec surface according to Newton's law of cooling, $\partial T^{*} / \partial n^{*}=\alpha_{t h}\left(T_{1}^{*}-T^{*}\right)$, where $n^{*}$ is the coordinate along the outward normal, $\alpha_{t h}$ is a phenomenological constant, and $T_{1}^{*}$ is the ambient temperature. In addition, we suppose that the density $\rho$ is constant (see the Appendix) and that the surface tension depends linearly on the temperature, $\sigma=\sigma_{0}-\gamma\left(T^{*}-T_{1}^{*}\right)$. In order to facilitate comparison with related results in the literature we nondimensionalize length, velocity, time and $T_{0}^{*}-T^{*}$ with $d^{*}, v / d^{*}, d^{* 2} / \nu$ and $T_{0}^{*}-T_{1}^{*}$, respectively, where $\nu$ is the kinematic viscosity. The resulting non-dimensional continuity, Navier-Stokes and energy equations in a reference frame attached to the bottom of the vibrating container $(z=0)$ are

$$
\begin{gathered}
\nabla \cdot \boldsymbol{u}+\partial_{z} w=0, \\
\partial_{t} \boldsymbol{u}+\boldsymbol{u} \cdot \nabla \boldsymbol{u}+w \partial_{z} \boldsymbol{u}=-\nabla p+\nabla^{2} \boldsymbol{u}+\partial_{z z}^{2} \boldsymbol{u}, \\
\partial_{t} w+\boldsymbol{u} \cdot \nabla w+w \partial_{z} w=-\partial_{z} p+\nabla^{2} w+\partial_{z z}^{2} w, \\
\partial_{t} \theta+\boldsymbol{u} \cdot \nabla \theta+w \partial_{z} \theta=\operatorname{Pr}^{-1}\left(\nabla^{2} \theta+\partial_{z z}^{2} \theta\right),
\end{gathered}
$$

for $(x, y) \in \Omega$ and $0<z<h(x, y, t)$. Here $\boldsymbol{u} \equiv(u, v, 0)$ and $w$ are the horizontal and vertical components of the velocity, respectively, $p\left(=\right.$ pressure $\left.+(G+a \omega)^{2} \cos \omega t\right)(z-$ 1)) represents a conveniently modified pressure, and $h$ is the (vertical) free-surface clcvation. In addition

$$
\nabla=\left(\partial_{x}, \partial_{y}, 0\right)
$$

denotes the horizontal gradient.

The boundary conditions at the supporting plate are no-slip, fixed temperature,

$$
\boldsymbol{u}=\mathbf{0}, \quad w=\theta=0 \quad \text { at } z=0,
$$

whilc at the free surface $z=h$ the kinematic boundary condition, tangential and normal stress balance, and heat flux balance, take the form

$$
\begin{aligned}
& \partial_{2} h+\boldsymbol{u} \cdot \nabla h=w, \\
& \partial_{z} \boldsymbol{u}+\nabla w-\left(\nabla \boldsymbol{u}+(\nabla \boldsymbol{u})^{-}\right) \cdot \nabla h+\left[2 \partial_{z} w-\left(\partial_{z} \boldsymbol{u}+\nabla w\right) \cdot \nabla h\right] \nabla h \\
& =\frac{M a}{P r}\left[\nabla \theta+\left(\partial_{\bar{z}} \theta\right) \nabla h\right], \\
& p-(\mathscr{G}+a \omega)^{2} \cos (\omega t)(h-1)+\mathscr{S} \nabla \cdot\left[\frac{\nabla h}{\left(1+|\nabla h|^{2}\right)^{1 / 2}}\right] \\
& =\frac{2 \partial_{z} w-2\left(\partial_{z} \boldsymbol{u}+\nabla w\right) \cdot \nabla h+\nabla h \cdot\left[\nabla \boldsymbol{u}+(\nabla \boldsymbol{u})^{\prime}\right] \cdot \nabla h}{1+|\nabla h|^{2}}, \\
& \partial_{z} \theta-\nabla h \cdot \nabla \theta=B i\left[1+|\nabla h|^{2}\right]^{1 / 2}(1-\theta) .
\end{aligned}
$$

$\Lambda$ s latcral boundary conditions, we consider two possibilitics. (a) If lateral walls are present, we impose no-slip and no-thermal-flux boundary conditions at these walls, and suppose that the contact line is either pinned or free, namely

$$
\boldsymbol{u}=\mathbf{0}, \quad w=\partial_{n} \theta=0, \quad \text { and either } \quad h=1 \text { or } \partial_{n} h=0
$$


for $(x, y) \div \partial \Omega$. IIere $\partial \Omega$ is the boundary of the cross-section $\Omega$ and $n$ is a coordinate along the outward unit normal. (b) In laterally unbounded films we consider periodic boundary conditions, namely

$$
\left.\begin{array}{l}
(\boldsymbol{u}, w, p, \theta)\left(x+L_{1}, y, z, t\right) \equiv(\boldsymbol{u}, w, p, \theta)\left(x, y+L_{2}, z, t\right) \equiv(\boldsymbol{u}, w, p, \theta)(x, y, z, t), \\
h\left(x+L_{1}, y, t\right) \equiv h\left(x, y+L_{2}, l\right)=h(x, y, t),
\end{array}\right\}
$$

and suppose that $L_{1} \gg 1, L_{2} \gg 1$. For convenience we also consider the vertically integrated continuity cquation,

$$
\partial_{2} h+\nabla \cdot\left(\int_{0}^{h} u \mathrm{~d} z\right)=0
$$

obtained from cquation (2.1) using (2.7).

The above problem depends on the following non-dimensional parameters: the Prandtl number $\operatorname{Pr}=\nu / \kappa$, where $\kappa$ is the thermal diffusivity, the Marangoni number $M a=\gamma\left(T_{0}^{*}-T_{1}^{*}\right) d^{*} / \rho \nu \kappa$, the Biot number $B i=\alpha_{t h} d^{*} / \kappa$, and the forcing amplitude $a=a^{*} / d^{*}$ and frequency $\omega=\omega^{*} d^{* 2} / \nu$. The gravitational and surface tension parameters, $\mathscr{G}=g d^{* 3} / \nu^{2}$ and $\mathscr{P}=\sigma_{0} d^{*} / \rho \nu^{2}$, are related to the usual Bond and Ohnesorge numbers, $B O=\rho g d^{* 2} / \sigma_{0}$ and $O h=v\left\lfloor\rho / \sigma_{0} d^{*}\right\rfloor^{1 / 2}$ by $\mathscr{G}=B O O h^{-2}$ and $\mathscr{T}=O h^{-2}$, where $g$ is the gravitational accelcration; $C=O h^{2}$ is called the capillary (or crispation) number.

The conduction state of the problem (2.1) (2.10), namely

$$
\boldsymbol{u}=\mathbf{0}, \quad w=0, \quad p=0, \quad \theta=\frac{B i z}{1+B i}, \quad h=1,
$$

exhibits two kinds of instabilities: a short-wave instability and a long-wave instability. Only the latter will be analysed below. The former could be called a Marangoni Bénard-Faraday instability and its study requires a numcrical solution of a Floquel problem, as done by $\$$ karda (2001) under the restriction that the free surface remains undeformed. For fixed $P r$, this instability appears only for quite large $M a$, namely $M a \sim 100$ (Bragard \& Velarde 1998; Skarda 2001). In addition, if the free-surface deformation is taken into account we have a Faraday instability (Kumar \& Tuckerman 1995; Mancebo \& Vega 2002). When

$$
1+\mathscr{G}^{2 / 3} \ll \omega,
$$

this instability can be avoided provided one chooses

$$
a \omega^{1 / 2} \equiv a^{*}\left(\omega^{*} / \nu\right)^{1 / 2}<A\left(\omega \mathscr{S}^{-2}\right) \equiv A\left(\omega^{*} \rho^{2} \nu^{3} / \sigma_{0}^{2}\right)
$$

where $A(\cdot)$ is a function calculated in Mancebo \& Vega (2002), shown in figure 1. Note in particular that the marginal instability curve has the horizontal asymptote $A \rightarrow A_{c:} \simeq 1.67$ as $\omega \rightarrow \infty$.

In what follows we shall assume that these short scales remain stable. 'lhis assumption is compatible with the distinguished limit

$$
\operatorname{Pr} \sim B i \sim M a \sim \mathscr{G} \sim 1, \quad \mathscr{f}^{1 / 2} \sim L \gg 1,
$$

where $L$ denotes the aspect ratio of the containcr. Since $G$ must be at most of order unity in order to avoid strong stabilization by gravity waves (see below) we replace (2.15) by the slightly more general requirement

$$
\omega \gg 1 \text {, }
$$




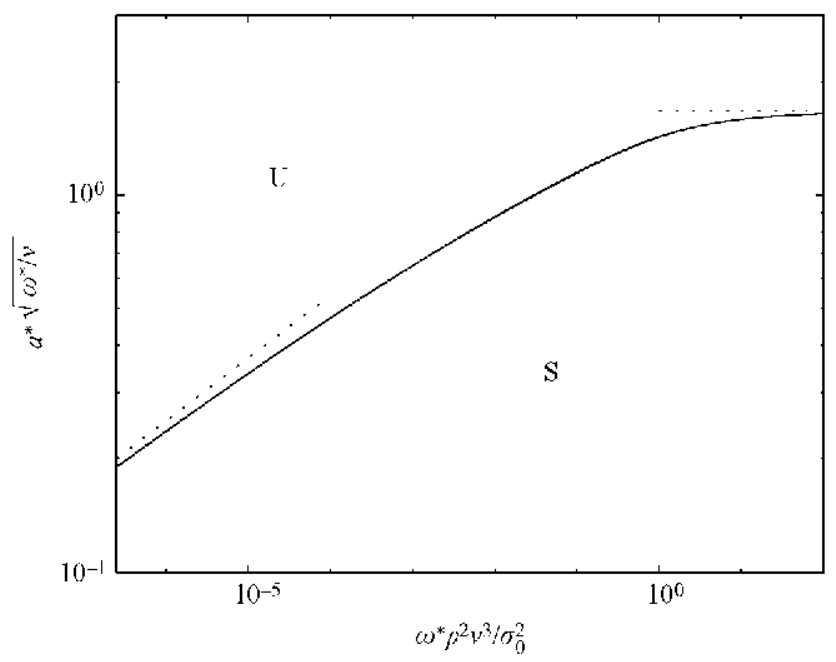

FIGURE 1. The rescaled l'araday instability threshold $\left.a \omega^{1 / 2}=a^{*}(\omega)^{*} / \nu\right)^{1 / 2}$ as a function of $\omega \mathscr{F}^{-2}=\omega^{+} \rho^{2} v^{3} / \sigma_{0}^{2}$ (see equation (2.16)). Stable (unstable) regions are labelled by $\mathrm{S}$ (U), respectively. The asymptotes for $\omega \mathscr{T}^{-2} \rightarrow 0$ and $\omega_{\mathscr{S}^{-2}} \rightarrow \infty$ are indicated by dotted lines. Courtesy of F. J. Mancebo.

subject to the constraint (2.16). In fact, the analysis below remains valid for arbitrary valucs of $B i$ and $\mathscr{A}$, provided that $\omega^{1} \ll \operatorname{Pr} \ll L^{2}, M a / \operatorname{Pr} \ll \omega L$ and $\mathscr{G} \ll \omega^{2} L^{2}$, restrictions that are quite reasonable even though $\operatorname{Pr}$ varies in practice over a wide range, from 0.01 for liquid metals to 105 for some silicone oils.

\section{Derivation of the long-wave equation}

The analysis in this section is a generalization of that by Lapuerta et al. (2001) who used vertical vibration to control the Raylcigh-'Taylor instability in large-aspect-ratio containers. In the limit (2.17)-(2.18) the main simplifications result from $(a)$ the longwave approximation and $(b)$ the fact that the problem exhibits two well separated timescales. In the regime

$$
\tilde{\mathscr{P}} \equiv \frac{\mathscr{F}}{L^{2}} \sim 1 \quad \text { and } \quad V i \equiv \frac{a^{2} \omega^{2}}{L^{2}} \sim 1
$$

we may introduce the slow space and time variables

$$
\tilde{x} \equiv \frac{x}{L}, \quad \tilde{y} \equiv \frac{y}{L}, \quad \tilde{t} \equiv \frac{t}{L^{2}},
$$

and seek a solution in the form

$$
\begin{aligned}
& \boldsymbol{u}=\frac{a \omega}{L} \boldsymbol{u}_{o}(\tilde{x}, \tilde{y}, z, \tilde{t}) \mathrm{e}^{\mathrm{i} \omega t}+\text { c.c. }+\frac{a^{2} \omega}{L^{3}} \mathrm{~h} . \mathrm{o} . \mathrm{h} .+\frac{1}{L} \boldsymbol{u}_{s}(\tilde{x}, \tilde{y}, z, \tilde{t})+\cdots, \\
& w=\frac{a \omega)}{L^{2}} w_{o}(\tilde{x}, \tilde{y}, z, \tilde{t}) \mathrm{e}^{\mathrm{i} \omega t}+\text { c.c. }+\frac{a^{2} \omega}{L^{4}} \text { h.o.h. }+\frac{1}{L^{2}} w_{s}(\tilde{x}, \tilde{y}, z, \tilde{t})+\cdots, \\
& p=a \omega^{2} p_{o}(\tilde{x}, \tilde{y}, z, \tilde{t}) \mathrm{c}^{\mathrm{i} \omega t}+\text { c.c. }+\frac{a^{2} \omega^{2}}{L^{2}} \text { h.o.h. }+p_{s}(\tilde{x}, \tilde{y}, z, \tilde{t})+\cdots, \\
& 0=\frac{a}{L^{2}} O_{o}(\tilde{x}, \tilde{y}, z, \tilde{t}) \mathrm{e}^{\mathrm{i} \omega t}+\text { c.c. }+\frac{a^{2}}{L^{4}} \text { h.o.h. }+O_{s}(\tilde{x}, \tilde{y}, z, \tilde{t})+\cdots
\end{aligned}
$$




$$
h=\frac{a}{L^{2}} h_{o}(\tilde{x}, \tilde{y}, \tilde{t}) \mathrm{e}^{\mathrm{i} \omega t}+\text { c.c. }+\frac{a^{2}}{L^{4}} \text { h.o.h. }+h_{s}(\tilde{x}, \tilde{y}, \tilde{t})+\cdots,
$$

where c.c. denotes the complex conjugate and h.o.h. stands for higher-order oscillatory harmonics, depending on the fast time variable as $\mathrm{e}^{\mathrm{i} m \omega t}$, with $m \neq 0, \pm 1$.

Substitution of this expansion into cquations (2.1)-(2.10) now leads to a series of equations for the oscillatory and slowly varying parts of the solution, labelled with the subscripts $o$ and $s$, respectively. The oscillatory part is nearly inviscid outside two oscillatory boundary layers atlached to the bottom plate and the free surface, and is given by

$$
\begin{gathered}
\tilde{\nabla} \cdot \boldsymbol{u}_{o}+\partial_{z} w_{o}=0, \quad \mathrm{i} \boldsymbol{u}_{o}+\tilde{\nabla} p_{o}=\mathbf{0}, \quad \partial_{z} p_{o}=0, \\
\mathrm{i} \theta_{o}+\boldsymbol{u}_{o} \cdot \tilde{\nabla} \theta_{s}+w_{o} \partial_{z} \theta_{s}=0 \quad \text { in } \quad 0<z<h_{s} \\
w_{o}=0 \quad \text { at } z=0 \\
\partial_{z} \boldsymbol{u}_{o}=\mathrm{i} h_{o}-w_{o}+\boldsymbol{u}_{o} \cdot \tilde{\nabla} h_{s}=p_{o}-\frac{h_{s}-1}{2}=0, \quad \partial_{z} \theta_{o}=-B i \theta_{o} \quad \text { at } z=h_{s}, \quad(3.10 a, b, c, d)
\end{gathered}
$$

where $\widetilde{\nabla}^{2}$ and $\widetilde{\nabla}$ are the rescaled horizontal Laplacian and gradient operators,

$$
\widetilde{\nabla}^{2}=\partial_{\tilde{x} \tilde{x}}^{2}+\partial_{\tilde{y} \tilde{y}}^{2} \quad \text { and } \quad \widetilde{\nabla}=\left(\partial_{\tilde{x}}, \partial_{\tilde{y}}, 0\right)
$$

The slowly varying part is given by

$$
\begin{aligned}
& \tilde{\nabla} \cdot \boldsymbol{u}_{s}+\partial_{z} w_{s}=0, \quad \partial_{z z} \boldsymbol{u}_{s}-\tilde{\nabla} p_{s}=V i\left[\left(\boldsymbol{u}_{o} \cdot \tilde{\nabla}\right) \boldsymbol{u}_{o}+\bar{w}_{o} \partial_{z} \boldsymbol{u}_{o}+\text { c.c. }\right], \\
& \partial_{z} p_{s}=\partial_{z z}^{2} \theta_{s}=0 \quad \text { in } \quad 0<z<h_{s}, \\
& \boldsymbol{u}_{s}=\mathbf{0}, \quad w_{s}=\theta_{s}=0 \quad \text { at } z=0, \\
& \partial_{\bar{t}} h_{s}-w_{s}+\boldsymbol{u}_{s} \cdot \widetilde{\nabla} h_{s}=p_{s}-\frac{V i}{2}\left(h_{o}+\text { c.c. }\right)-\mathscr{G}\left(h_{s}-1\right)+\mathscr{\mathscr { T }} \widetilde{\nabla}^{2} h_{s}=0 \text {, } \\
& \partial_{z} \boldsymbol{u}_{s}=\frac{M a}{P_{r}}\left[\widetilde{\nabla} \theta_{s}+\left(\partial_{z} \theta_{s}\right) \widetilde{\nabla} h_{s}\right], \quad \partial_{z} \theta_{s}-B i\left(1-\theta_{s}\right)=0 \text { al } z=h_{s}, \\
& \left(\int_{0}^{h_{s}} \boldsymbol{u}_{s} \mathrm{~d} z\right) \cdot \boldsymbol{n}=0 \text { and either } h_{s}=1 \text { or } \partial_{n} h_{s}=0 \text { for }(\tilde{x}, \tilde{y}) \in \partial \tilde{\Omega} \text {, }
\end{aligned}
$$

if lateral walls are present. For unbounded films the boundary conditions (3.15) must be replaced by periodic boundary conditions. Oscillatory viscous boundary layers contribute $O\left(\omega^{-1}\right)$ corrections to the right-hand sides of the boundary conditions $(3.13 a)$ and $(3.14 c)$; since $\omega \gg 1$ (see $(2.18))$ these corrections are small. In addition, the presence of lateral walls requires a zero fluid flux outside $O\left(L^{-1}\right)$ boundary layers attached to the walls; the analysis of these layers is standard and is omitted.

If we neglect the effects of both types of boundaries layers (sec Nicolás \& Vega 2003 and references therein) and solve equations (3.8)-(3.14) we obtain

$$
\begin{gathered}
\theta_{s}=z H, \quad p_{o}=\frac{1}{2}\left(h_{s}-1\right), \quad \mathbf{u}_{o}=\frac{\mathrm{i}}{2} \tilde{\nabla} h_{s}, \quad w_{o}=-\frac{\mathrm{i} z}{2} \widetilde{\nabla}^{2} h_{s}, \quad h_{o}=-\frac{1}{2} \tilde{\nabla} \cdot\left(h_{s} \tilde{\nabla} h_{s}\right), \\
p_{s}=-\frac{V i}{2} \tilde{\nabla} \cdot\left(h_{s} \widetilde{\nabla} h_{s}\right)+\mathscr{G}\left(h_{s}-1\right)-\tilde{\mathscr{F}} \widetilde{\nabla}^{2} h_{s}, \\
\boldsymbol{u}_{s}=\frac{1}{2}\left(z^{2}-2 z h_{s}\right) \tilde{\nabla}\left(p_{s}+\frac{V i}{4}\left|\widetilde{\nabla} h_{s}\right|^{2}\right)+\frac{M a}{\operatorname{Pr}} z \tilde{\nabla}\left(h_{s} H\right)
\end{gathered}
$$


where

$$
I \equiv \frac{B i}{1+B i h_{s}} .
$$

The equation for $O_{0}\left(=-\frac{1}{2} z \tilde{\nabla} H \cdot \tilde{\nabla} h_{s}+\frac{1}{2} z H \widetilde{\nabla}^{2} h_{s}\right)$ decouples.

The first boundary condition in (3.14) is cquivalent to the continuity equation

$$
\partial_{i} h_{s}+\tilde{\nabla} \cdot\left(\int_{0}^{h_{s}} \boldsymbol{u}_{s} \mathrm{~d} z\right)=0
$$

an cquation that can also be obtained from (2.13). Substituting for $\boldsymbol{u}_{s}$ yiclds the following evolution equation for $h_{s}$ :

$$
\partial_{\bar{t}} h_{s}=\widetilde{\nabla} \cdot\left[\frac{1}{3} h_{s}^{3} \widetilde{\nabla}\left(p_{s}+\frac{V i}{4}\left|\widetilde{\nabla} h_{s}\right|^{2}\right)-\frac{M a}{2 P r} h_{s}^{2} \widetilde{\nabla}\left(h_{s} I I\right)\right] .
$$

Dropping the subscript $s$ from $h$ and tildes from $\nabla$, and introducing the new parameters

$$
V=\frac{V i}{2 \tilde{\mathscr{G}}} \equiv \frac{\rho a^{* 2} \omega^{* 2} d^{*}}{2 \sigma_{0}}, \quad G=\frac{\mathscr{G}}{\mathscr{G}} \equiv \frac{\rho g L^{* 2}}{\sigma_{0}}, \quad M=\frac{M a}{\operatorname{Pr} \mathscr{G}} \equiv \frac{\gamma\left(T_{0}^{*}-T_{1}^{*}\right)}{\rho g d^{* 2}},
$$

and the rescaled time variable

$$
\tau=\tilde{F} \tilde{t}
$$

cquation (3.19) becomes

$$
\partial_{\tau} h=-\frac{1}{3} \nabla \cdot\left\{h^{3} \nabla\left[\nabla^{2} h+V\left(h \nabla^{2} h+\frac{1}{2}|\nabla h|^{2}\right)-f^{\prime}(h)\right]\right\},
$$

where

$$
f(h)=G \frac{h^{2}}{2}-\frac{3}{2} G M B i h \ln \frac{h}{1+B i h} .
$$

This cquation is to be solved subject to the boundary conditions (3.15):

$$
\partial_{n}\left[\nabla^{2} h+V\left(h \nabla^{2} h+\frac{1}{2}|\nabla h|^{2}\right)-f^{\prime}(h)\right]=0,
$$

and either $h=1$ or $\partial_{n} h=0$ for $(x, y) \in \partial \Omega$.

\section{Analysis of the long-wave equation for a horizontal substrate}

\subsection{Linear stability}

In an unbounded domain the flat film, $h=1$, is linearly stable when

$$
\frac{3 M B i}{2(1+B i)^{2}}<1 \text {. }
$$

This condition is independent of $V$; in view of (3.20) it is also independent of viscosity. Moreover, since $M$ is proportional to $M a / G$ and $G$ is quite large for thick films under terrestrial conditions (Davis 1987), gravity waves tend to stabilize the film unless it is highly viscous or quite thin. For instance, in a $1 \mathrm{~mm}$ deep silicone oil film with $v=0.01 \mathrm{~cm}^{2} \mathrm{~s}^{1}$ we have $\mathscr{G}=10^{4} ; \mathscr{G}$ falls to 1 if $\nu=1 \mathrm{~cm}^{2} \mathrm{~s}^{1}$ or $d^{*}$ is decreased to $50 \mu \mathrm{m}$.

For bounded containers condition (4.1) must be replaced by

$$
\frac{3 M B i}{2(1+B i)^{2}}<1+s_{0} \frac{V+1}{G} .
$$




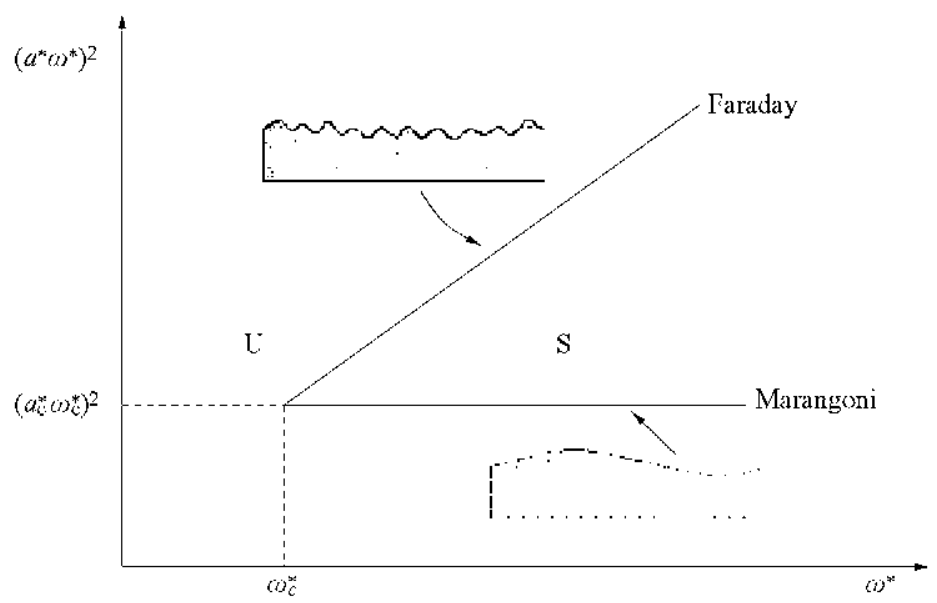

FIGURE 2. A typical linear stability diagram for the flat-film solution (equation (2.14)), indicating regions of stability (S) and instability (U). The critical parameters $\omega_{c}^{*}$ and $a_{c}^{*}$ are defined by the crossing of the long-wave Marangoni and Faraday instability thresholds. Stability with respect to the short-wave Marangoni instability is assumed.

where $s_{0}$ is the lowest eigenvalue of

$$
\widetilde{\nabla}^{2} F+s F=\text { constant in } \tilde{\Omega}, \quad \text { and either } F=0 \text { or } \partial_{n} F=0 \text { on } \partial \tilde{\Omega} .
$$

For instance, for a circular container of unit radius $s_{0}=\gamma_{1} \simeq 3.83$ or $s_{0}=\gamma_{2} \simeq 2.40$ depending on the boundary condition, where $\gamma_{1}$ and $\gamma_{2}$ are the first positive roots of $J_{1}$ (the Bessel function of order one) and $J_{1}^{\prime}$, respectively. If $\tilde{\Omega}$ is a $1 \times D$ rectangle, $s_{0}=\pi^{2}\left(1+4 / D^{2}\right)$ or $s_{0}=4 / D^{2}$.

Thus in a finite domain the stability condition does depend on $V$. Moreover, since $V$ is proportional to the scaled vibration energy $\left(a^{*} \omega^{*}\right)^{2}$, equation (4.2) shows that for a given value of the remaining parameters the system is stable provided that $\left(a^{*} \omega^{*}\right)^{2}$ is sufficiently large, subject to the restriction (2.16). The resulting stability region rescmbles that shown in figure 2. Note that the lower curve is independent of viscosity while the upper curve moves downwards and to the right as viscosity decreases. Thus the forcing frequency for stabilization must be quite large for lowviscosily liquids.

For containers that are large compared to the wavelength of the fastest linear mode one may disregard the boundary conditions at the walls of the container and study the behaviour of spatially periodic solutions. This allows comparison with results obtained for heated thin films without vibration (Thiele \& Knobloch 2004). 'The stability condition is given by equation (4.2) replacing $s_{0}$ by $k^{2}$ with $k$ being the wavenumber of the mode under study. The critical wavenumber is given by

$$
k_{c}^{2}=\frac{G}{1+V}\left(\frac{3 M B i}{2(1+B i)^{2}}-1\right),
$$

while the growth rate $\beta$ of wavenumber $k$ is

$$
\beta=-\frac{1+V}{3} k^{2}\left(k^{2}-k_{c}^{2}\right)
$$

Thus at the linear level the stabilizing role of vibration mimics enhanced surface tension. This enhancement is measured by $V$ and so is again independent of 
viscosity; however, viscosity comes into play through condition (2.16). For instance, this condition is satisfied under microgravity conditions by a container of depth $5 \mathrm{~mm}$ filled with silicone oil with $\sigma=20 \mathrm{dyn} \mathrm{cm}^{-1}, \nu=0.01 \mathrm{~cm}^{2} \mathrm{~s}^{-1}$ and $\rho=1 \mathrm{~g} \mathrm{~cm}^{-3}$, subject to vibration amplitude $a^{*}=5 \mu \mathrm{m}$ and frequency $\omega^{*}=10^{4} \mathrm{~Hz}$; for these parameter values $V=60$. For higher viscosity liquids we can use lower vibration frequency and obtain an even larger enhancement. For instance, for $v=1 \mathrm{~cm}^{2} \mathrm{~s}^{-1}, a^{*}=0.5 \mathrm{~mm}$ and $\omega^{*}=2 \times 10^{2} \mathrm{~Hz}$ condition (2.16) remains satisfied but $V=200$.

\subsection{Lyapunov functional}

The vibration docs not break the variational structure (Oron et al. 1997) of the static counterpart of equation (3.22). Indeed, if we define a Lyapunov functional 6 by

$$
=\frac{1}{2} \int_{\Omega}\left[|\nabla h|^{2}+V h|\nabla h|^{2}+f(h)\right] \mathrm{d} x \mathrm{~d} y,
$$

where $f(h)$ is defined by (3.23), it is simple to show that

$$
\frac{\mathrm{d} \mathscr{G}}{\mathrm{d} \tau}=-\frac{1}{3} \int_{\Omega} h^{3}|\nabla \mathscr{q}|^{2} \mathrm{~d} x \mathrm{~d} y,
$$

where

$$
\not l l=\nabla^{2} h+V\left(h \nabla^{2} h+\frac{1}{2}|\nabla h|^{2}\right)-f^{\prime}(h) .
$$

The quantity $\mathscr{U}$ is defined such that equation (3.22) takes the form

$$
h_{\tau}=-\frac{1}{3} \nabla \cdot\left(h^{3} \nabla \text { भ }\right) .
$$

Thus every solution of equations (3.22) (3.23) converges for large times to a steady statc.

\subsection{Steady states}

Since the local energy $f(h)$ does not depend on the vibration it is now possible to draw on the results of 'Thiclc \& Knobloch (2004) regarding the nonlinear stability of a flat film. Fquation (4.7) implies that the steady states of (3.22)-(3.24) are given by

$$
\begin{gathered}
\nabla^{2} h+V\left(h \nabla^{2} h+\frac{1}{2}|\nabla h|^{2}\right)-f^{\prime}(h)+C_{1}=0 \quad \text { in } \Omega, \\
\int_{\Omega} h \mathrm{~d} x \mathrm{~d} y=S_{\Omega 2} \text { and either } h=1 \text { or } \partial_{n} h=0 \quad \text { if }(x, y) \in \partial \Omega,
\end{gathered}
$$

where $S_{\delta 3}$ is the area of $\Omega$, and $C_{1}$ is a constant corresponding to the Lagrange multiplier for either volume conservation or a chemical potential depending on the situation studicd. Equation (4.10) can also be obtained from equation (3.22) sctting $\partial_{\tau} h=0$ and using condition (3.24).

In particular, in one dimension, the steady states are given by

$$
h^{\prime \prime}+V\left[h h^{\prime \prime}+\frac{1}{2} h^{\prime 2}\right]-f^{\prime}(h)+C_{1}=0,
$$

or, equivalently, by

$$
\frac{1}{2}(1+V h) h^{\prime 2}-f(h)+c_{1} h+c_{2}=0,
$$

where $C_{2}$ is another constant. The two constants $C_{1}, C_{2}$ characterize the steady-state solutions. Spccifically,

$$
\begin{aligned}
& C_{1}=-\frac{1}{2} V o_{i}^{2}+f^{\prime}\left(h_{i}\right), \\
& C_{2}=f\left(h_{m}\right)-C_{1} h_{m},
\end{aligned}
$$



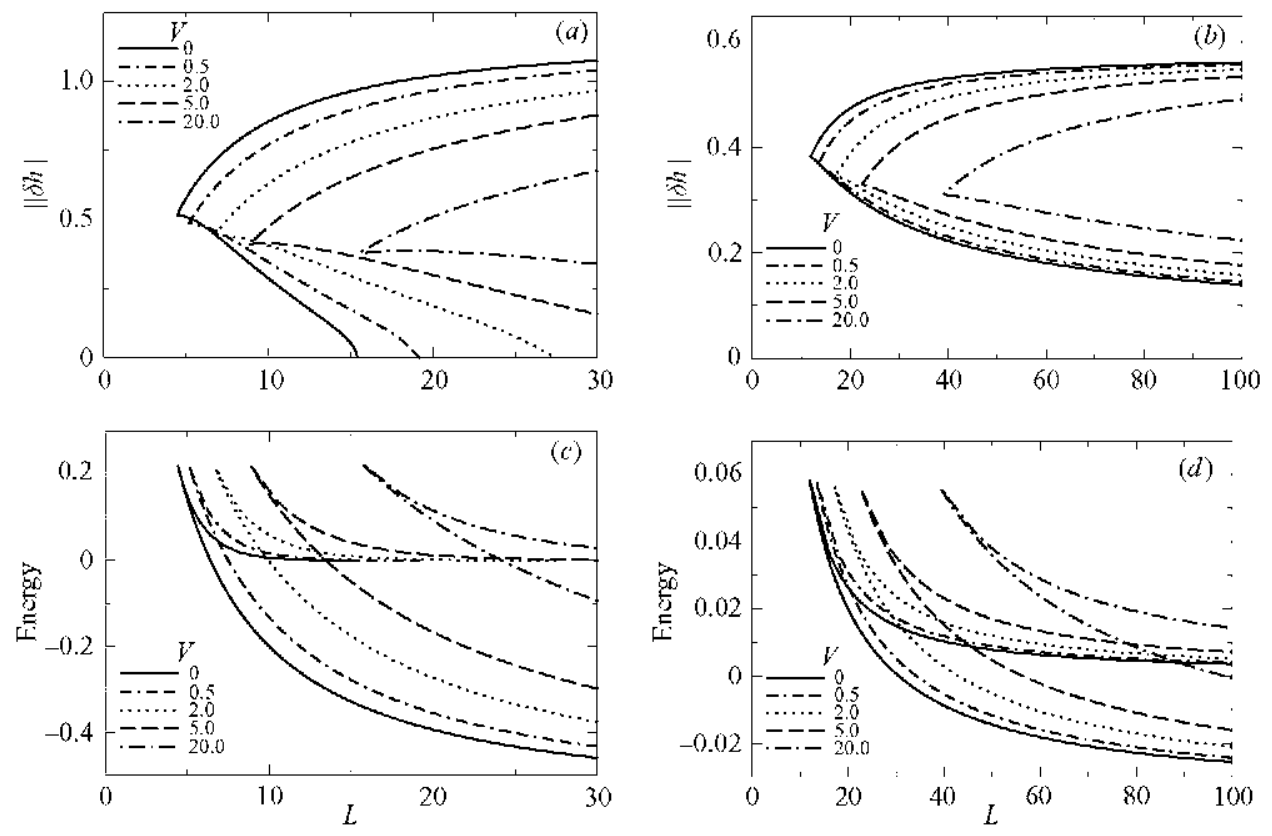

Figure 3. Periodic nucleation and single-drop solutions with microscopic contact angle $\theta_{0}=0$ as a function of $L$ for $B i=0.5$ and $G=1.0$ and $(a, c) M=3.5>M_{c}$ (linearly unstable regime) and $(b, d) M=1.5<M_{c}$ (the metastable regime). (a) and (b) the norm for the nucleation solutions (Iower branches) and the drop solutions (upper branches). These meet at cusps located at $\left(L^{*}, h^{*}\right)$. Stable single-drop solutions with $\theta_{0}>0$ are found in the region above the upper branch. $(c)$ and $(d)$. The corresponding relative energies per unit length $E(L)$. Proliles for the nucleation and drop solutions are shown in figure 5.

where $h_{i}$ and $h_{m}$ denote the film thickness at the inflection point and maximum thickness, respectively. The angle $\theta_{i} \equiv\left|h_{i}^{\prime}\right|$ measures the slope of the film at the inflection point and for drop-like solutions is identificd with the so-called mesoscopic contact angle. It is this angle and not the microscopic contact angle $\theta_{0}$ at $h=0$ that is normally measured in expcriments.

Tquation (4.13) must be integrated using the boundary conditions (4.11), namely

$$
\bar{h} \equiv L^{1} \int_{0}^{L} h(x) \mathrm{d} x=1 \quad \text { and either } h=1 \text { or } h^{\prime}=0 \quad \text { at } \quad x=0, L .
$$

The integral constraint represents mass conservation, and provides an implicit relation between the constants $C_{1}, C_{2}$, or equivalently the quantities $h_{i}, h_{m}$. The solutions with Neumann boundary conditions can be obtained from the solutions on a periodic domain with period $2 L$; however, this is not so for Dirichlet boundary conditions and more generally for Robin (or mixed type) boundary conditions (Crawford et al. 1991).

In the following we study periodic stationary solutions, described by cquation (4.13). To facilitate comparison with the vibration-free case (Thiele \& Knobloch 2004) we fix the $x$-scale $\ell=\sigma_{0} / \rho G$ by setting $G=1$. This leaves the period of the stationary solutions as a free parameter. Note that the $B i$ and $M$ used here correspond to the $B i$ and $M a$ of Thiele \& Knobloch (2004), while $G=1.0$ corresponds to $B o=1.0$.

Since $h=1$ the periodic solutions can be parametrized using the film volume (or, in one dimension, its spatial period $L$ ). Thus for each parameter combination $(M, B i, V)$ there is a one-parameter family of solutions. Higure 3 shows this family 
for different $V$ as a function of the imposed spatial period $L$ in terms of the norm $|\delta h| \equiv\left(\int_{0}^{L}(h(x)-1)^{2} \mathrm{~d} x / L\right)^{1 / 2}$ (figure $\left.3 a, b\right)$ and the relative energy per unit length $E=\mathscr{C} / L-f(1)$ (figure $3 c, d)$. Figure $3(a)$ shows the norm when the flat film is unstable $(B i=0.5, M=3.5)$ : a one-parameter family of steady solutions bifurcates subcritically from the flat film for which $|\delta h|=0$. 'I'he solutions on the subcritical branch are all unstable, and we refer to them as nucleation solutions. This is because an initial perturbation with a smaller amplitude (and $h=1$ ) will decay to the flat film, while one with larger amplitude will evolve to a drop-like state on the upper branch (see below). A similar statement holds for sliding isothermal drops (Thiele et al. 2001). The branch of nucleation solutions terminates at a finitc valuc of $L, L=L^{*}$, where the minimum thickness drops to zero. The upper branch emerging from $L^{*}$ corresponds to drop-like solutions with microscopic contact angle $\left.\theta_{0} \equiv h^{\prime}\right|_{h=0}=0$ sitting on a dry substrate. Above this branch one can find a large variety of drop-like states with $0_{0}>0$, as discussed further in Thiele \& Knobloch (2004). As shown in figure 3(c) the cnergy $E$ of the nuclcation solutions is always larger than that of the flat film for which $E_{0}=0$. Moreover, the figure also indicates that there is a small range of periods above $L^{*}$ where the flat film has the lowest energy. Thus even though the fixed-period drop solutions correspond to local minima of the energy (i.e. they are linearly stable) they do not necessarily represent global minima. As shown in figures $3(b)$ and $3(d)$ for $B i=0.5$ and $M=1.5$ the nucleation solutions may be present even when the flat film remains stable for all $L$, but the corresponding solution branch is now disconnected from the flat-film solution. We say in this case that the flat-film state is metastable.

'The 'potential' $g(h)=f(h)-C_{1} h$ with $C_{1}=f^{\prime}(1)$ (shown for $B i=0.5$ and various $M$ in figure 3 of Thiele \& Knobloch 2004) determines whether the flat film $(h=1)$ is unstable, metastable or stable. l'he boundary between the unstable and metastable regimes is given by $g^{\prime \prime}(1)=0$, and corresponds to the linear stability result (4.1). The transition from metastable to stable corresponds to parameter valucs for which the minima of $g(h)$ at $h=1$ and $h=0$ are identical, i.e. to $g(1)=0$. This transition therefore occurs along the curve

$$
M_{m s}=\frac{1+B i}{3 B i}
$$

a quantity that is also independent of $V$. The resulting stability diagram in the $(B i, M)$-plane is shown in figure 4. In particular, in the limit $B i \gg 1$ the flat film is unstable, metastable and stable for $M>2 \mathrm{Bi} / 3,2 \mathrm{Bi} / 3>M>1 / 3$ and $M<1 / 3$, respectively. However, metastable drop solutions may exist even below $M_{m s}$. Figure 5 shows examples of nucleation and drop solutions for different values of $V$ and fixed volume. With increasing vibration the droplets become flatter, a result that resembles that obtained for isothermal pendant and sitting droplets by Gavrilyuk et al. (2004).

The above results indicate that vibration stabilizes the flat film against instability and quantify the ellect. Specifically, we have secn that for increasing $V$ :

(i) the threshold for linear instability of the film is shifted towards larger wavelength, i.e. system size (see equation (4.4) and figure 6);

(ii) the range of existence of the drop solutions is shifted towards larger periods, i.e. smaller drops do not exist any more (see the location of the cusp $\left(L=L^{*}\right)$ in figure 3 and figure 6);

(iii) the boundary between metastable and stable flat films shifts towards larger system size; 


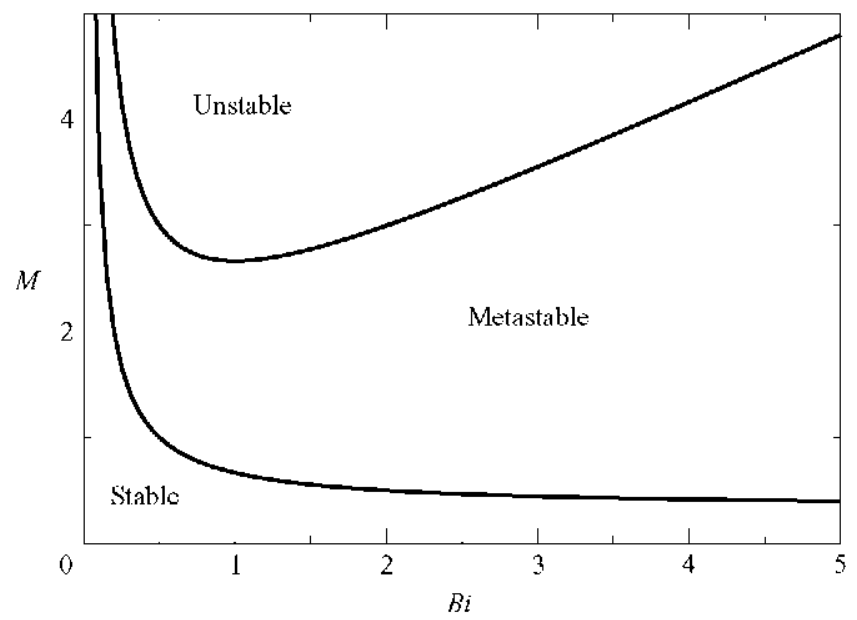

Figure 4. The stability of a liat infinitely extended film in the $(B i, M)$-plane for $(i=1.0$, showing regions of stability, metastability and instability. The location of the three regions is independent of $V$.
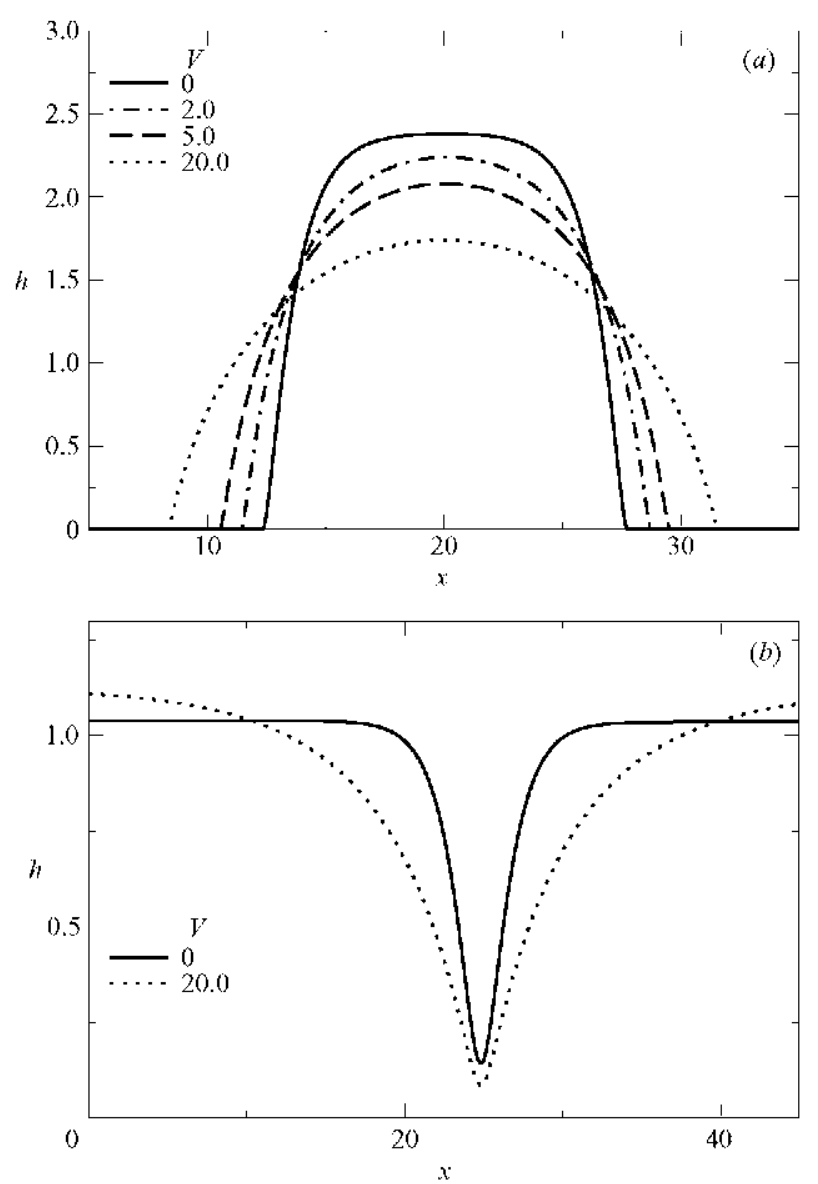

FIGURE 5. Solution profiles for $B i=0.5, G=1.0$, fixed volume $L$ and different values of $V$. (a) Drop solutions for $L=30, M=3.5$, (b) nucleation solutions for $L=100, M=1.5$ (only part of the period is shown). 


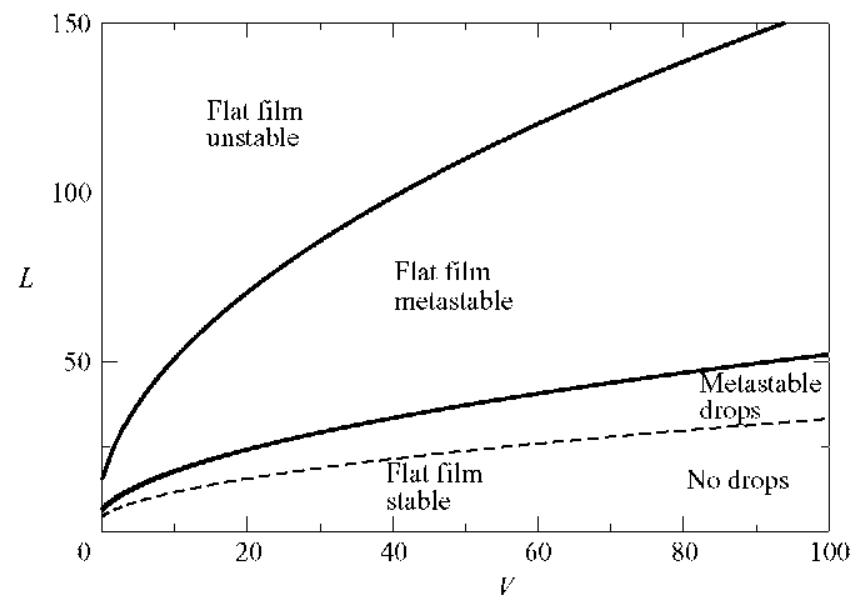

Figure 6. The stability of a liat film in a linite system of size $L$ as a function of $V$ for $B i=0.5$ and $M=3.5$. In the stable range a subrange where no drops exist and one where drops are metastable ean be distinguished. The boundary between the two subranges corresponds to the locus of the saddle-nodes in figure $3(a)$, while the boundary between stable and metastable flat films corresponds to loci of the zero crossing of $E$ in figure $3(\mathrm{c})$. The curves are all of the form $L=2 \pi \chi \sqrt{1+V}$ with $\chi=0.52,0.83$ and $\sqrt{6} \approx 2.45$, respectively.

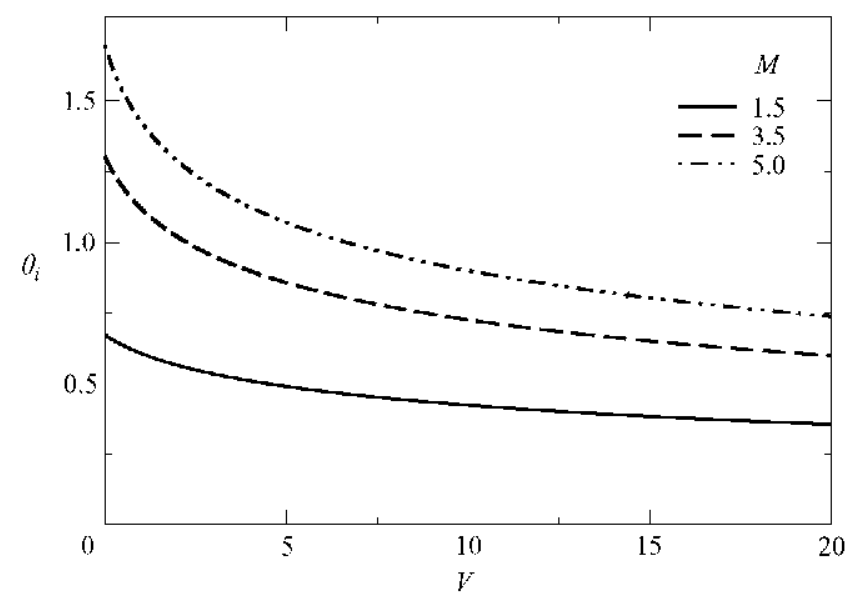

Figidre 7. The mesoscopic contact angle $O_{i}$ as a function of $V$ for different values of $M$ when $B i=0.5, G=1.0$ and $L=100$.

(iv) the amplitude (i.e. the maximum height) of the drops decreases, as does the norm (sec figures 3 and $5 a$ );

(v) the mesoscopic contact angle $\theta_{i}$ decreases as illustrated by the drop profiles in figure 5. The dependence of $O_{i}$ on $V$ is shown for different $M$ in figure 7;

(vi) for metastable flat films the nucleation solution (the critical dent) that has to be overcome to generate instability becomes deeper and broader, as illustrated in figure $5(b)$;

(vii) for a fixed volume of liquid (i.e. the spatial period $L$ ) the energy $E$ (figure 3 ) of the drop increases with increasing $V$, indicating that the drop absorbs part of the 

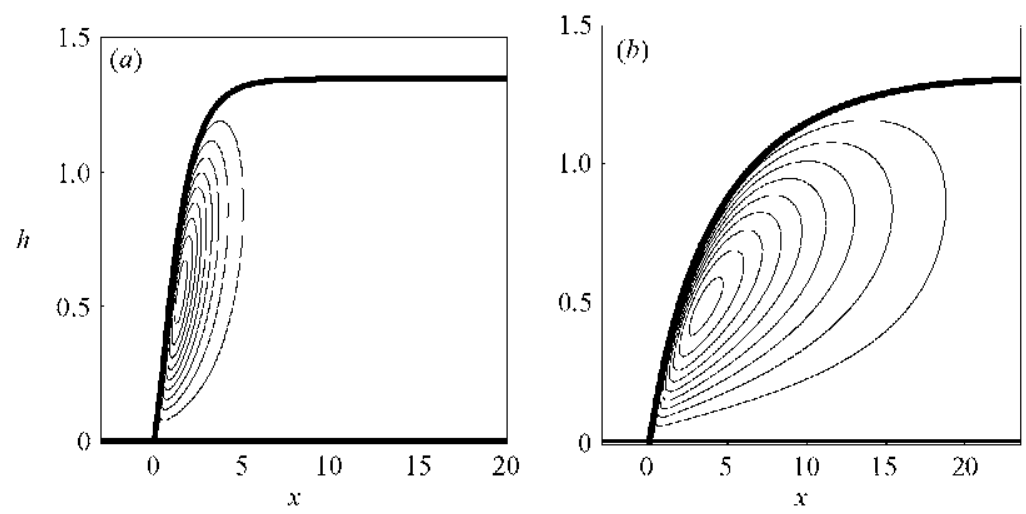

FIGURE 8. Streamlines $\psi$ of the convection pattern inside a drop on a dry substrate for $B i=0.5, G=1.0, M=1.5$ and $L=50$. (a) $V=0, \psi=-4 i \times 10^{-4},(b) V=20, \psi=-i \times 10^{-4}$, for $i=1 \ldots 9$. The fluid rises near the drop surface and descends within the drop. The drop profile itself corresponds to $\psi=0$.

vibration energy. This energy is not dissipated but goes into modifying the shape of the drop (see below).

It is instructive to examine the corresponding changes in internal convection within the drops. We do so using contour lines of the streamfunction $\psi$ given by

$$
\psi(x, z)=\left(\frac{z^{2} h}{2}-\frac{z^{3}}{6}\right)\left\lceil h^{\prime \prime \prime}-G h^{\prime}+V\left(2 h^{\prime} h^{\prime \prime}+h h^{\prime \prime \prime}\right)\right]+\frac{z^{2}}{2} \frac{M G B i h^{\prime}}{(1+B i h)^{2}} .
$$

Figure 8 shows that in the presence of vibration the circulation penctrates much decper into the drop, even though it is in fact weaker. As a result the overall dissipation for $V=20$ is approximately half of the dissipation for $V=0$.

We remark that the presence of the factor $L \gg 1$ relating the horizontal and vertical coordinates in equations (3.2) and (3.7) implies that all 'pairwise' comparisons, such as $\partial_{x x} u \ll \partial_{z z} u$, used to simplify the Navicr-Stokes equation in the long-wave approximation are satisfied; a direct comparison of $u$ and $w$ is not required for the validity of this approximation, and closed streamlines, such as those in figure 8 , do not violate the conditions required by the theory. In particular, the long-wave approximation remains valid in a moving reference frame, used in the following section to study the influcnec of vibration on a flowing film on a slightly inclined substrate.

\section{Thin film on an inclined plane}

\subsection{Stationary states}

When the substrate is slightly inclined in the $x$-direction, a driving term due to gravitational acceleration has to be incorporated in equation (3.22), yiclding

$$
\partial_{\tau} h=-\frac{1}{3} \nabla \cdot\left\{h^{3} \nabla\left[\nabla^{2} h+V\left(h \nabla^{2} h+\frac{1}{2}|\nabla h|^{2}\right)-f^{\prime}(h)\right]\right\}-\alpha h^{2} \partial_{x} h .
$$

IIere $\alpha / \ell$ is the inclination of the substrate relative to the horizontal; $f(h)$ is unchanged and defined as in (3.23). 
Stationary solutions in one dimension in a frame moving downstream with velocity v therefore satisfy the equation

$$
\frac{h^{3}}{3}\left\{(1+V h) h^{\prime \prime \prime}+2 V h^{\prime} h^{\prime \prime}-f^{\prime \prime}(h) h^{\prime}+\alpha\right\}-v h+c_{0}=0 .
$$

Here $h$ depends only on the comoving variable $\xi=x-v t$, and $C_{0}$ is a constant of integration that, in contrast to the reflection-symmetric case $\alpha=0$, cannot be set to zero. The constant $C_{0}$ may be identified with the flux of liquid in the comoving systcm. We choose

$$
C_{0}=-\frac{1}{3} h_{0}^{3} \alpha+v h_{0} \equiv \Gamma_{0}+v h_{0},
$$

corresponding to a uniform film of thickness $h_{0}$. The corresponding flux in the laboratory frame is then given by $\Gamma_{0}=-h_{0}^{3} \alpha / 3$. Note that for a given value of $\Gamma_{0}$ there may be a second homogeneous solution with the same flux, given by

$$
\tilde{h}_{0}=h_{0}\left(-\frac{1}{2}+\sqrt{\frac{3 v}{\alpha h_{0}^{2}}-\frac{3}{4}}\right) .
$$

where $h_{0}$ specifies the value of $\Gamma_{0}$. Such a solution is present whenever $v / \alpha h_{0}^{2}>1 / 3$, and allows for shock or front solutions. It corresponds to the so-called conjugate solution, as discussed in the falling film context, for instance, by Chang (1989) and Nguyen \& Balakotaiah (2000).

Spatially periodic uniformly travelling solutions of equations (5.2), (5.3) can be found analytically for small amplitudes and computed using continuation (Doedel et al. 1997) in either the period $L$ or one of the other dimensionless parameters of the problem. $\Lambda$ s before we fix the mean film thickness to $h=1$ implying a dependence of the constant $C_{0}$ on the other parameters. The resulting nonlinear solutions solve a nonlincar cigenvalue problem for the speed $v$ specified by equation (5.2) subject to periodic boundary conditions with period $L$.

To solve this problem in the weakly nonlinear regime we let $h=1+\epsilon \delta_{0}+\epsilon^{2} \delta_{1}+$ $c^{3} \delta_{2}+\cdots$, where $0<c \ll 1$ and the $\delta_{j}, j \geqslant 0$, have zero mean in order to ensure mass conservation. We must also expand $v$ and $C_{0}$ in powers of $\epsilon: v=v_{0}+\epsilon v_{1}+\epsilon^{2} v_{2}+\ldots$, $C_{0}-v+(\alpha / 3)=\epsilon^{2} K_{2}+\ldots$. Thus $K_{2}$ represents a correction to the llux required by mass conservation.

$\Lambda \mathrm{t} O(\epsilon)$ we obtain the linear problem

$$
\mathscr{L} \delta_{0} \equiv \frac{1}{3}(1+V) \delta_{0}^{\prime \prime \prime}-\frac{1}{3} f^{\prime \prime}(1) \delta_{0}^{\prime}+\left(\alpha-v_{0}\right) \delta_{0}=0 .
$$

Here primes on the variable $\delta_{0}$ indicate derivatives with respect to $\xi$ whilc those on $f$ indicate derivatives with respect to $h$ and evaluated at $h=1$. Fquation (5.5) is solved by $\delta_{0}=a \sin k \xi$, where

$$
(1+V) k^{2}+f^{\prime \prime}(1)=0, \quad v_{0}=\alpha .
$$

This equation determines the threshold for the instability. For example, in a periodic domain of length $L$ we must have $k=k_{n} \equiv 2 \pi n / L, n=1,2, \ldots$ In the following we shall be interested in values of $L$ close the corresponding critical values $L_{n}=2 \pi n \sqrt{-(1+V) / f^{\prime \prime}(1)}, n=1,2, \ldots$, namcly $L=L_{n}+\epsilon^{2} \mu$.

At next order we solve the problem

$$
\mathscr{L} \delta_{1}=-\left(1+\frac{4}{3} V\right) \delta_{0} \delta_{0}^{\prime \prime \prime}-\frac{2}{3} V \delta_{0}^{\prime} \delta_{0}^{\prime \prime}+\left(f^{\prime \prime}(1)+\frac{1}{3} f^{\prime \prime \prime}(1)\right) \delta_{0} \delta_{0}^{\prime}-\alpha \delta_{0}^{2}+v_{1} \delta_{0}-K_{2} .
$$


Thus

$$
\delta_{1}=a^{2}(A \sin 2 k \xi+B \cos 2 k \xi), \quad v_{1}=0, \quad K_{2}=-\frac{1}{2} a^{2} \alpha,
$$

where

$$
A=\frac{\alpha}{4 k f^{\prime \prime}(1)}, \quad B=\frac{1}{4 f^{\prime \prime}(1)} \frac{V f^{\prime \prime}(1)-\frac{1}{3}(1+V) f^{\prime \prime \prime}(1)}{1+V} .
$$

Finally, at $O\left(\epsilon^{3}\right)$, we have to solve the problem $\mathscr{L} \delta_{2}=N_{2}\left(\delta_{0}, \delta_{1}, \mu\right)$. The solvability conditions at this order yield

$$
\begin{gathered}
-\frac{2}{3 L}(1+V) k^{2} \frac{\mu}{a^{2}}=\left(\frac{7}{2}+4 V\right) k^{2} B+\frac{1}{2}\left(f^{\prime \prime}(1)+\frac{1}{3} f^{\prime \prime \prime}(1)\right) B+\frac{A}{k} \alpha-\frac{3}{4} V k^{2} \\
-\frac{1}{4}\left(f^{\prime \prime \prime}(1)+\frac{1}{6} f^{\mathrm{iv}}(1)\right), \\
\frac{v_{2}}{a^{2}}=\left(\frac{7}{2}+4 V\right) k^{3} A+\frac{1}{2}\left(f^{\prime \prime}(1)+\frac{1}{3} f^{\prime \prime \prime}(1)\right) k A+\left(\frac{1}{4}-B\right) \alpha .
\end{gathered}
$$

Here $k=k_{n} \equiv 2 \pi n / L_{n}$, and

$$
\begin{aligned}
f^{\prime \prime}(1) & =G-\frac{3 G M B i}{2(1+B i)^{2}}, \quad f^{\prime \prime \prime}(1)=\frac{3 G M B i(1+3 B i)}{2(1+B i)^{3}}, \\
f^{(i v)}(1) & =-\frac{3 G M B i\left(1+4 B i+6 B i^{2}\right)}{(1+B i)^{4}} .
\end{aligned}
$$

We can think of equation (5.10) as an equation for $\mu$ given $a$. It follows that if $\mu>0$ the bifurcation is supercritical, while if $\mu<0$ it is subcritical. The case $\mu=0$ is therefore the transition case and corresponds to

$$
\frac{\alpha^{2}}{k_{n}^{2}}=\frac{1}{2}\left(k_{n}^{2}+f^{\prime \prime}(1)-\frac{1}{3} f^{\prime \prime \prime}(1)\right)^{2}+\frac{1}{6} f^{\prime \prime}(1) f^{\mathrm{iv}}(1) .
$$

This condition can be viewed as an equation for either $M$ or $V$ or indeed $\alpha$; its predictions agrec well with numerical computations, as discussed next.

These solutions can be extended into the fully nonlinear regime using numerical continuation. This is most easily done by starting from the neutrally stable solutions of the lincarized problem, i.c. sinusoidal solutions with period $L_{c}=2 \pi / k_{c}$ where $k_{c}$ is given by equation (4.4), or equivalently by equation (5.6). We may start from a solution consisting of just one period $L_{c}$ or from a 'replicated' solution with period $n L_{c}$, where $n$ is an integer. We call the resulting solution branches emanating from the zero amplitude state (uniform film) n-mode primary branches. Branches bifurcating from these in secondary bifurcations are called sccondary solution branches. 'the $n>1$ primary branches can be obtained directly from the $n=1$ branch by multiplying the solution period by $n$. Solutions on such branches have 'internal symmetry' $Z_{n}$, in addition to the $S O(2)$ symmetry due to translation invariance of the system. The secondary bifurcations either respect the discrete $Z_{n}$ symmetry (in which case they correspond to saddle-node bifurcations) or break it, creating a secondary branch of lower symmetry.

We focus here on the $n=1$ primary branches, a selection of which is shown in figure 9. Fach panel shows curves for different values of $V$ for a fixed inclination angle $\alpha$ and $G=1.0, B i=0.5, M=3.5$. The figure shows that increasing $V$ at fixed $\alpha$ changes the bifurcating branches and the corresponding film profiles in a way that resembles qualitatively the changes found for $V=0$ when $\alpha$ increases. Thus the presence of vibration does not lead to qualitatively new types of behaviour. 

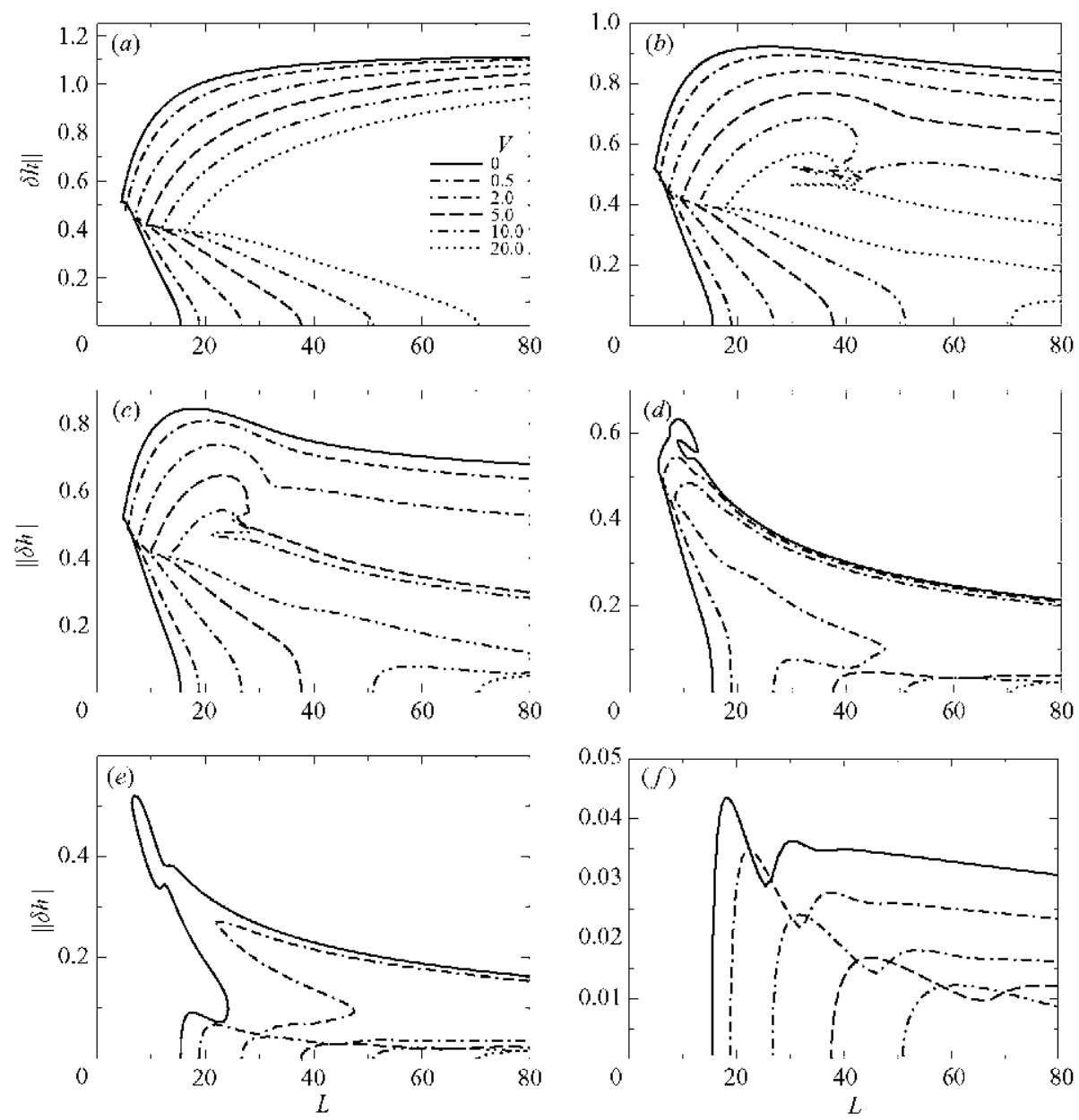

FIGURE 9. The $n=1$ solution branches obtained by continuation from small-amplitude solutions for different values of $\alpha$ and $V$ when $G=1.0, B i=0.5, M=3.5$, and $(a) \alpha=0.01$, (b) $0.07,(c) 0.1,(d) 0.19$, (e) $0.29,(f) 0.5$. (a-f) The values of $V$ correspond to the legend in $(a)$.

For small $\alpha$ (figure $9 a$ ) an increase in $V$ has a similar effect as on the horizontal substrate, namely the vibration decreases the $L_{2}$ norm of the profiles. Figures $10(a)$ and $10(b)$ show that as the vibration number increases for fixed $\alpha$ the drop profile begins to resemble a spherical cap. At the same time the internal circulation penetrates much deeper into the drop. Moreover, the drift velocity $v$ of both nucleation and drop solutions of fixed period decreases with increasing vibration (figure 11a), primarily becausc oscillations in the mesoscopic contact angle tend to pin the state in place. Ilowever, the situation for the surface wave states present at larger inclinations $\alpha$ is quite different (see below), largely because for these states the mesoscopic contact angle is unimportant.

Provided $\alpha$ is not too large (figure $9 a e$ ) an increase in $V$ induces a transition from large-amplitude sliding drop solutions to small-amplitude surface waves. This reflects the transition from a Cahn-I Iilliard-type dynamics to a Kuramoto-Sivashinsky-type dynamics described by Thiele \& Knobloch (2004), and is accompanied by a change in 

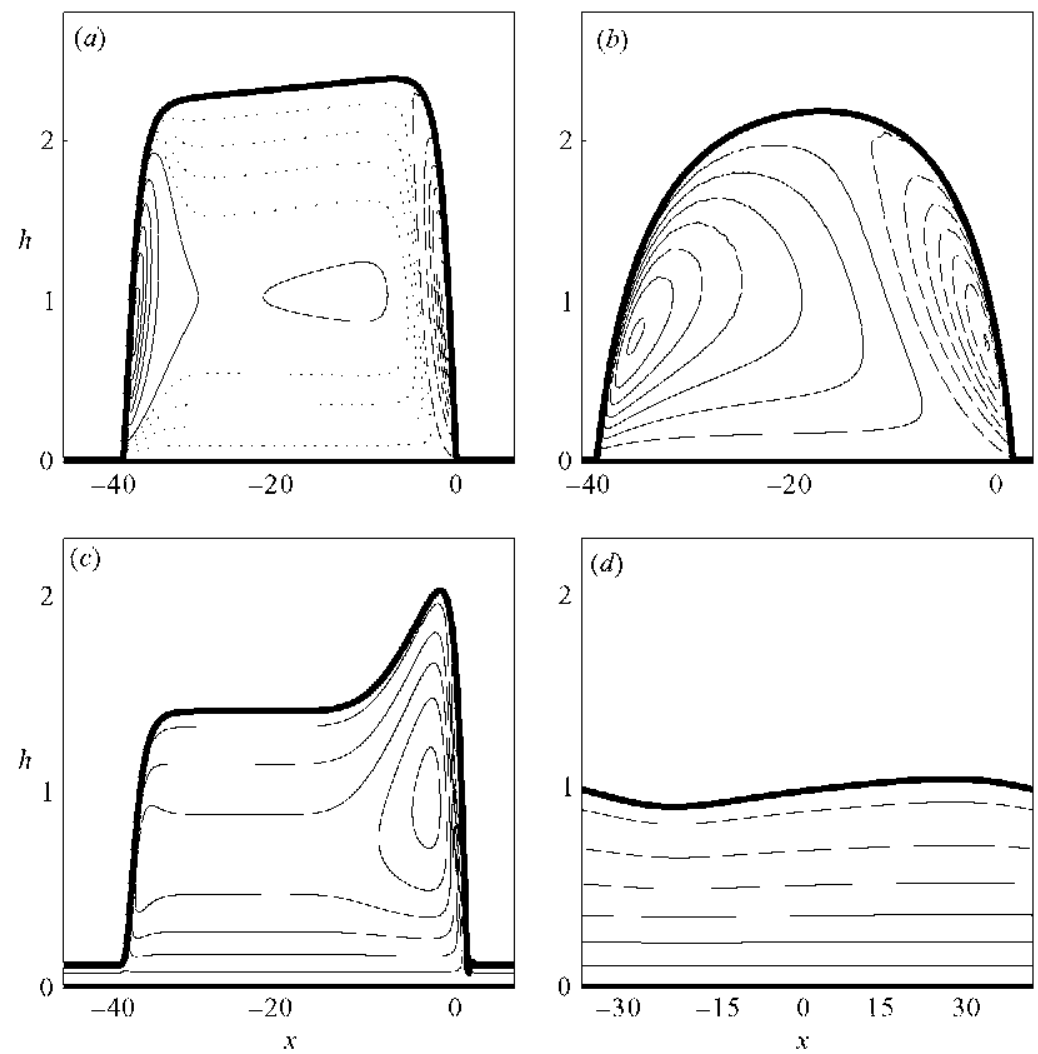

FIGiJRe 10. Drop profiles with associated streamlines in the comoving frame. $(a, b) \alpha=0.01$, and $(c, d) \alpha=0.1$. 'The parameters are $L=80, G=1.0, B i=0.5, M=3.5$ and $V=0$ for $(a, c)$, and $V=20$ for $(b, d)$. 'The streamline intervals and drop velocities $v$ are $(a) \Delta \psi=0.005$, $v=0.011,(b) \Delta \psi=0.005, v=0.0063,(c) \Delta \psi=0.005, v=0.072$, and $(d) \Delta \psi=0.01, v=0.099$. Solid (dashed) streamlines represent clockwise (counter-clockwise) flow; dotted lines in (a) show intermediate clockwise streamlines with $\Delta \psi=0.001$.

the character of the primary bifurcation from subcritical to supercritical at $V=V_{\text {crit }}$ given by equations (5.13) and (5.6). This dramatic change in the surface profile is accompanied by a similarly dramatic change in the flow pattern (cf. figures $10 \mathrm{c}$ and $10 d)$. In the absence of vibration $(V=0)$ the drop is flat, with a capillary ridge at the leading front, above a pair of strongly asymmetric convection rolls; behind the capillary ridge the streamlines are nearly parallel, while at the back of the drop there is again a stronger upward flow driven by the surface tension gradient. In contrast, when $V$ is increased to $V=20$ the drops are replaced by a freely flowing film with a small surface undulation and streamlines that are almost parallel (figure 10d). This state is similar to the surface wave states present at larger inclinations when $V=0$.

For large $\alpha$ (figure $9 f$ ) surface waves dominate already for $V=0$. As $V$ increases their amplitude decreases while their spatial period increases. Since the amplitude of the surface deflection is reduced, vibration makes the film less unstable. In addition the drift velocity $v$ increases with increasing vibration (figure $11 b$ ) largely because it augments the effective gravitational accelcration acting on these states.

The overall change from drop-like solutions to surface waves with increasing inclination or vibration strength can be captured by focusing on the loci of the 

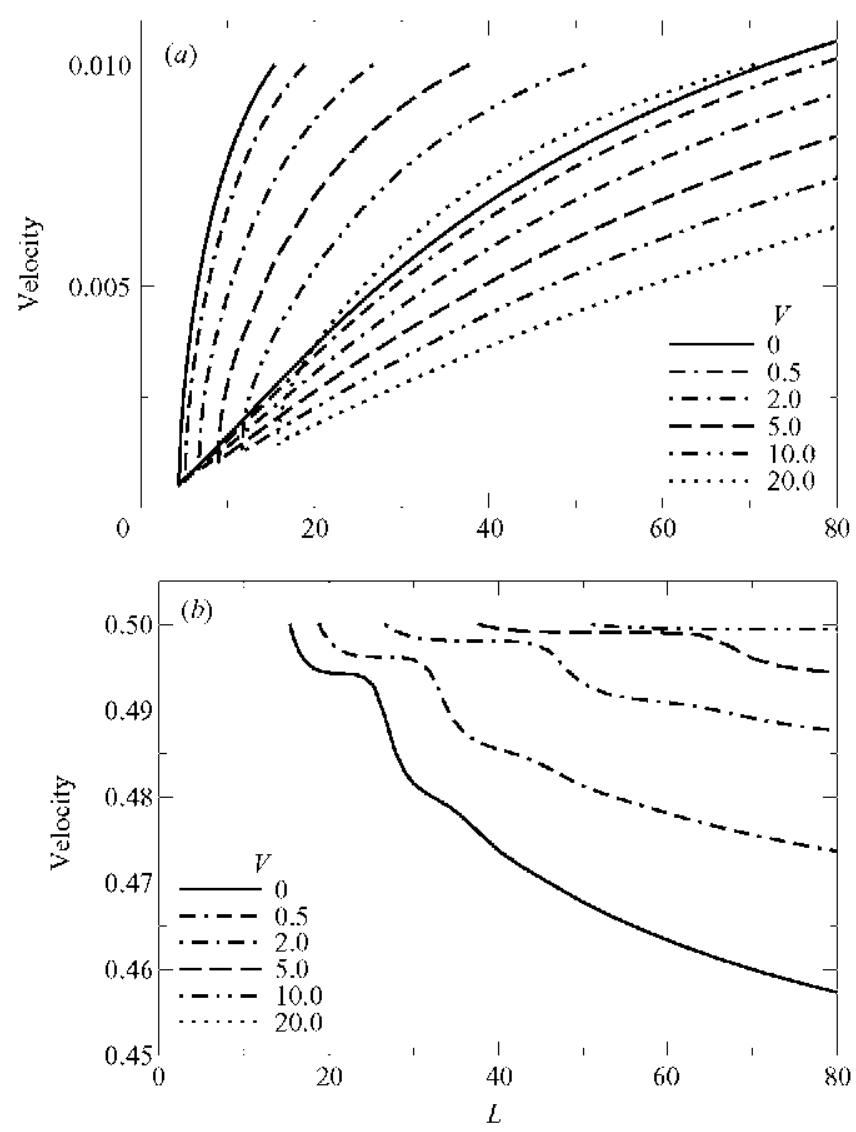

Figiure 11. The drilt velocity along the $n=1$ solution branch for diflerent values of $V$ and (a) $\alpha=0.01$ (nucleation states above, drop states below), (b) $\alpha=0.5$ (surface waves). The parameters are $G=1.0, B i=0.5, M=3.5$.

main saddle-node bifurcations and the change from sub- to supercritical primary bifurcation. Figure 12(a) shows these loci as a function of $V$ for diflerent $\alpha$ whereas figure 12(b) compares the analytical prediction (5.13) for the transition from subcritical to supercritical primary bifurcation to the numcrical results. For very small inclinations, $\alpha=0.01$, only one saddle-node bifurcation is present, and this moves towards larger periods with increasing $V$. Thus the primary bifurcation is subcritical, and it remains so until $V \approx 760$. 'The figure shows that the distance (on a logarithmic scale) between the saddle-node and the primary bifurcation remains approximately constant. Thus the range of periods with unstable nucleation solutions increases with increasing $V$ (cf. figure $9 a$ ).

For larger inclinations, e.g. $\alpha=0.1$, the primary bifurcation becomes supercritical as indicated by the appcarance of the upper dashed line in figure 12(a). This line represents the loci of the right-most saddle-node bifurcations (cf. figure 9). In contrast, the locus of the left-most saddle-node bifurcation remains essentially unchanged. This implies that for larger $V$ there is a large range in $L$ where stable surface waves and drop-like states coexist. However, as $\alpha$ increases yet more this coexistence range shrinks drastically. For example, for $\alpha=0.19$ the transition from sub- to supcreritical primary bifurcation occurs at $V \approx 0.8$, indicating the appearance of surface waves, while the left-most saddle-node bifurcation moves off to infinity when $V \approx 6$. No 

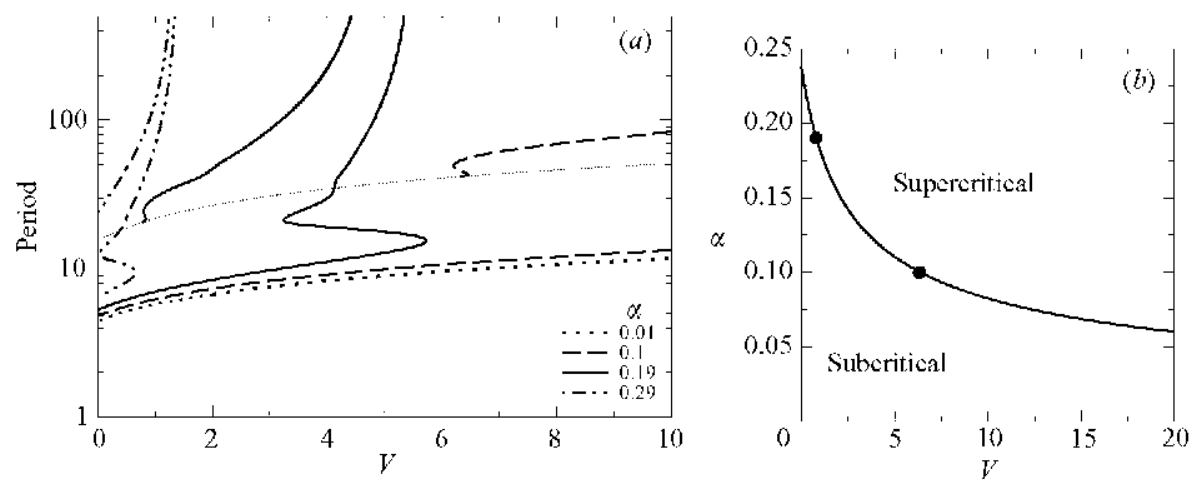

FIGURE 12. (a) Loci of the main saddle-node bifurcations on the $n=1$ primary branch in the $(V, L)$-plane for different inclinations $\alpha$. The thin dotted line denotes the primary instability of the flat film solution. (b) The transition from sub- to supercritical primary bifurcation in the $(V, \alpha)$-plane obtained analytically (equations (5.13) and (5.6)). The solid circles correspond to the numerical results shown in (a). The paramelers are $G=1.0, B i=0.5, M=3.5$.

drop-like states can exist beyond this value of $V$. The oscillation in this curve reflects the formation of an isola of solutions: at $V \approx 3.2$ an isola detaches from the primary branch near the left-most saddle-node in a reverse 'necking' bifurcation. The resulting loop of stationary states shrinks and vanishes at $V \approx 5.7$.

At even larger inclinations, $\alpha=0.29$, the primary bifurcation is always supercritical (cf. figure 9e), and the upper dot-dashed line in figure 12(a) no longer reaches the thin dotted line. $\Lambda$ isola is present at small $V$, but only surface waves are present for $V \gtrless 1.8$.

\subsection{Linear stability}

We next turn to the lincar stability propertics of the stationary solutions obtained in $\$ 5.1$ for the inclined substrate. Because we are interested in the overall influence of vibration we restrict our attention to the $n=1$ primary branch, i.e. we do not study instabilitics lcading to coarsening; for the latter we would have to investigate the stability properties of $n>1$ primary branches as well.

To determine the stability properties of the stationary nonlinear solutions $h_{0}(\xi)$ of equation (5.2) obtained in $\$ 5.1$ we write $h(\xi)=h_{0}(\xi)+c h_{1}(\xi) \mathrm{e}^{\beta \tau}$, where $h_{1}$ is an infinitesimal perturbation of $h_{0}$ in the frame moving with the velocity $v$. Equation (5.1) in one dimension, linearized in $\epsilon$, yiclds an eigenvalue problem for the growth rate $\beta$ and the associated eigenfunction $h_{1}(\xi)$ :

$$
\beta h_{1}=L\left(h_{0}, h_{0}^{\prime}, h_{0}^{\prime \prime}, h_{0}^{\prime \prime \prime}, h_{0}^{\prime \prime \prime}\right) h_{1}
$$

where $L$ is a fourth-order linear differential operator acting on the perturbation $h_{1}(\xi)$ whose coefficients depend nonlinearly on $h_{0}$ and its spatial derivatives.

The solution of this problem is again based on continuation techniques. The cigenvalues and cigenfunctions are obtained in a threc-step procedure. First, we determine $h_{0}$ as described in $\$ 5.1$. Second, the eigenvalue problem is discretized in space using equidistant discretization and solved numerically. However, this technique is severely limited in its applicability because it lacks accuracy for large periods or steep profiles $h_{0}(\xi)$ and, more generally, for small eigenvalues $\beta$. To avoid these difficultics we employ in a third step numcrical continuation of the solution of the nonlinear eigenvalue problem (5.2) together with the linear eigenvalue problem (5.14). The complete system used in the third step therefore consists of eleven first-order 

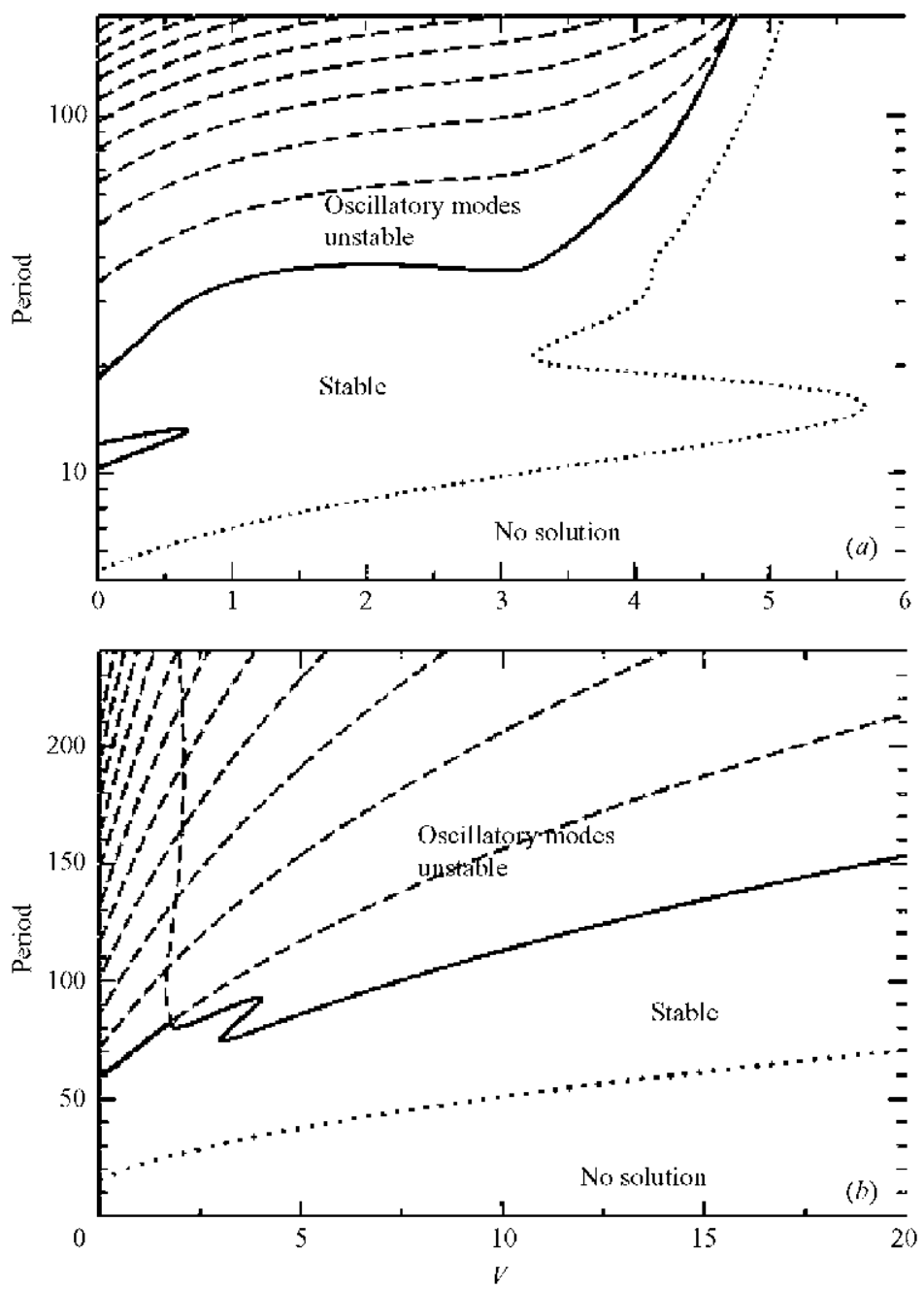

FIgiJRe 13. The linear stability of $n=1$ stationary solutions of dillerent periods as a function of the vibration number $V$ for $(a) \alpha=0.19$ and $(b) \alpha=0.4$. 'The solid lines delimit the instability region (shaded) and correspond to stationary instability in the comoving frame. 'l'he dashed lines indicate the location of subsequent oscillatory instabilities. The dotted line in $(a)$ indicates the locus of the saddle-node bifurcations at which the primary branch acquires stability, while the dotted line in $(b)$ corresponds to the primary (supereritical) bifureation. Thus in $(b) n=1$ solutions exist only above the dotted line. The parameters are $(i=1.0, B i=0.5, M=3.5$.

differential equations (three for $h_{0}$ and four for the real and imaginary parts of $h_{1}$, respectively). Using this procedure with the result of step two as a starting point we can calculate both $h_{0}$ and $h_{1}$, and the associated eigenvalues $v$ and $\beta$, in parallel for any system parameters. Moreover, points of special interest, such as the location of zero growth rate (i.e. bifurcation points), can also be followed through parameter space.

$\Lambda$ s a general tendency we find that increasing the vibration number $V$ stabilizes the stationary solutions. Jowever, there are small ranges of $V$ in which the opposite happens. We illustrate these conclusions in figure 13 computed for two inclinations $\alpha$. 
Figure $13(a)$, for $\alpha=0.19$, summarizes the linear stability properties on the upper part of the $n=1$ primary branch (cf. figure $9 d$ ). The branch is stable with respect to real modes but becomes unstable to a variety of oscillatory modes as $L$ increases. When $V=0$ there is a small unstable region near $L=10$; thereafter the branch is stable up to an instability threshold $L_{c} \approx 20$. $\Lambda$ s $L$ increases, more and more oscillatory modes become unstable. Thus the branch is unstable for $L>L_{t ;}$. As $V$ increases the instability interval near $L \approx 10$ starts to shrink and cventually vanishes when $V \approx 0.7$. The instability threshold $L_{c}$ initially moves towards larger periods leaving a larger range of stable solutions. However, between $V \approx 2$ and $V \approx 3$ the threshold shifts slightly towards smaller $L$, decreasing the range of stable solutions. Once $V>3$ the stationary solutions correspond to surface waves and $L_{c:}$ moves rapidly towards larger pcriods as $V$ increascs, indicating that vibration has a strong stabilizing influcnce on these states. At $L \approx 250$ and $V \approx 4.9$ the instability thresholds for different modes start to cross, with the fourth oscillatory mode eventually becoming dominant. It is this mode that destabilizes the high- $L$ part of the $n=1$ branch once $V \geq 5.2$.

Figure $13(b)$ shows the corresponding results for $\alpha=0.4$. In this case all states are surface waves. 'The figure shows that these states are always stable with respect to real modes but become unstable to oscillatory ones as $L$ increases. The instability threshold $L_{c}$ is determined by two different oscillatory modes. At small $V, V<1.7$, the instability is dominated by a mode that is unstable even when $V=0$. In fact, there is a very small range of $V, 0<V<0.1$, where $L_{c}$ decreases with increasing $V$ (from 62.5 to 61.3). For $V>1.7$ a different mode takes over that is not present when $V=0$. Between $V \approx 3$ and $V \approx 4$ a small but finite range of stable periods exists near $L=80$, but for larger $V$ the threshold $L_{t}$ increases monotonically, confirming again that vibration has a generally stabilizing influence.

\section{Concluding remarks}

In this paper we have derived, using lubrication theory, an evolution equation describing the effect of high-frequency vertical oscillation on a thin film on either a horizontal substrate or a slightly inclined one. The equation incorporates, in addition to vibration, thermocapillary effects, hydrostatic and Laplace pressure, and gravitational driving when the substrate is inclined, and represents a new thin-film equation. The equation was derived under the assumption that short-wave Faraday instability is absent, and conscquently that the only modes of interest are long wave. In addition we assumed that the short-wave Marangoni instability (Golovin, Nepomnyashchy \& l'ismen 1994) is also absent. The former assumption is not very restrictive; the latter restricts the practical application of our analysis to films that are not too thick. I Iowever, the film cannot be too thin either, in order to justify our omission of van der Waals interaction with the substrate. For films of thickness $100 \mathrm{~nm}$ or less the resulting disjoining pressure can be included in a straightforward fashion (Bestehorn et al. 2003). However, our theory applies to films this thin only for extremcly high vibration frequencies, $\omega^{*}=10^{8} \omega \mathrm{s}^{-1}$ for $h=100 \mathrm{~nm}$.

Our study of the resulting evolution equation revealed that normal vibration has in general a stabilizing influence. This is so for $(a)$ a flat film on a horizontal substrate, $(b)$ stationary drops on a horizontal substrate, (c) sliding drops on an inclined substrate, and $(d)$ surface wave states on an inclined substrate. In particular, vibration raises the threshold wavelength for the instability of flat films and decreases the mesoscopic contact angle of drops while smoothing their profile and decreasing their height (cf. figure 8). Vibration also moves the drop states to larger periods, i.e. a stationary 
Fluid

Density $\rho\left(\mathrm{kg} / \mathrm{m}^{3}\right)$

Kincmatic viscosity y $\left(10^{6} \mathrm{~m}^{2} / \mathrm{s}\right)$

Thermal diffusivity $\kappa\left(10^{-6} \mathrm{~m}^{2} / \mathrm{s}\right)$

Surlace tension gradient $\gamma=d \sigma / d T$ $\left(10^{-4} \mathrm{~kg} / \mathrm{s}^{2} \mathrm{~K}\right)$

Surlace tension $\sigma_{0}\left(\mathrm{~kg} / \mathrm{s}^{2}\right)$
H'I 70

1680

0.5

0.043

$\mathrm{NA}$

0.014
Silicon oil $5 \mathrm{cS}$

920

5.0

0.008

NA

0.0197
Silicon oil $10 \mathrm{cS}$

940

10.2

0.1

0.69

0.0201

TABı.E 1. Matcrial parameters for 5cS and 10cS silicon oil, and for HT70, taken from VanHook et al. (1997); Fngel \& Swift (2000); Jucl et al. (2000). HT70 is a perfluorinated hydrocarbon (also called a Galden fluid).

vibrating drop cannot exist if its volume is too small. I Iowever, the resulting drop-like states may be more subcritical than in the absence of vibration. At larger inclinations vibration can turn subcritical primary bifurcations into supercritical oncs, and generally makes such bifurcations more supercritical, thereby favouring surface waves over drop-like states. Thus vibration shifts the transition from Cahn Hilliard-type dynamics to Kuramoto-Sivashinsky-type dynamics described in Thiele \& Knobloch (2004) towards smaller inclination angles. At the same time vibration improves the mixing properties of Marangoni convection, particularly in large droplets, an effect that may have important consequences for drops consisting of binary mixtures where concentration effects can modify not only the surface tension but the static or dynamic contact angles as well.

At present there are no experimental studies of the influence of high-frequency normal vibration on long-wave Marangoni instability or on drop shape on a heated substrate. Even in the absence of vibration, experiments on the long-wave Marangoni instability of a thin liquid layer or the bchaviour of sitting drops on a heated horizontal substrate are relatively sparse (VanIIook et al. 1995, 1997). To our knowledge no experimental results are available on the dependence of the contact angle of sitting drops on substrate temperature or of the instability wavelength on the temperature and film thickness. Such investigations are worthwhile, however, and could be used to study the influence of high-frequency vibration. In the following we suggest a possible experiment of this type.

Table 1 lists material parameters for specific fluids used in existing Marangoni experiments. Typical values are $\kappa=0.05 \times 10^{-6} \mathrm{~m}^{2} \mathrm{~s}^{-1}$ (note that the Prandtl number can be large), $\rho=1000 \mathrm{kgm}^{3}, \sigma_{0}=0.02 \mathrm{kgs}^{2}$, and $\gamma=1.0 \times 10^{4} \mathrm{kgs}^{2} \mathrm{~K}{ }^{1}$. A typical range for the imposed temperature difference is $0.05<\delta T<5.0 \mathrm{~K}$, while the thickness of the flat film satisfies $50<d<250 \mu \mathrm{m}$ (VanIIook et al. 1995). The requirement that the parameter $G$ in equation (3.20) be equal to 1 (assumed here) leads to a value of the horizontal length $L^{*}=0.0015 \mathrm{~m}$. If we take $d^{*}=150 \mu \mathrm{m}$, then the requirement that $V \sim 1$ in equation (3.20) leads to $a^{*} \omega^{*}=0.55 \mathrm{~m} \mathrm{~s}{ }^{1}$. With these values the requirement that points below the marginal instability curve in figure 1 be stable to the Taraday instability leads to the condition that $a^{*} \sqrt{a^{*} / v} \leqslant 1$, requiring that $\omega^{*} v>3000$. Thus $\omega^{*} \rho^{2} v^{3} / \sigma_{0}^{2} \gg 100$. Typical parameter values leading to points in the Faraday-stable regime are then the following: $(a) v=0.003 \mathrm{~m}^{2} \mathrm{~s}^{-1}$, $\omega=100 \mathrm{~s}^{1} a^{*}=5.5 \mathrm{~mm} ;(b) \nu=0.0003 \mathrm{~m}^{2} \mathrm{~s}^{1}, \omega=1000 \mathrm{~s}^{1}, a^{*}=0.55 \mathrm{~mm}$; or $(c) v=$ $0.00003 \mathrm{~m}^{2} \mathrm{~s}^{-1}, \omega=10000 \mathrm{~s}^{-1}, a^{*}=0.055 \mathrm{~mm}$. If instcad, we require that $V \sim 20$ (as in many calculations in the paper), then $a^{*}(\omega) \sim 24 \mathrm{~m} \mathrm{~s}^{-1}$, and $\omega^{*} v$ must be greater than 60000 , which leads to $(a) \nu=0.06 \mathrm{~m}^{2} \mathrm{~s}^{1}, \omega=100 \mathrm{~s}^{1}, a^{*}=24 \mathrm{~mm} ;(b) \nu=0.006 \mathrm{~m}^{2} \mathrm{~s}^{1}$, 
$\omega=1000 \mathrm{~s}^{1}, a^{*}=2.4 \mathrm{~mm}$; or $(c) v=0.0006 \mathrm{~m}^{2} \mathrm{~s}^{1}, \omega=10000 \mathrm{~s}^{1}, a^{*}=0.24 \mathrm{~mm}$. We are aware that some of these combinations of $\omega^{*}$ and $a^{*}$ can be diflicult to attain in practice, but they provide an indication of the requirements of the theory. On the other hand, the requirement that $M \sim 1$ yiclds $T_{0}^{* *}-T_{1}^{*} \sim 2 \mathrm{~K}$, which agrees well with existing long-wave Marangoni experiments (VanI Iook et al. 1997).

The proposed experiments could be used on the one hand to study the influence of vibration on long-wave Marangoni instability in large-aspect-ratio domains, and on the other hand to study the characteristics of individual sitting and sliding drops under the influence of heating and vibration. In the former the interesting questions range from the suppression of the initial surface instability to the effect of vibration on long-time coarsening behaviour; in the latter case theory predicts that on an inclined substrate the transition from drop-like states to surface waves should be very sensitive to vibration.

The thin-film model incorporating vibration derived here can be extended to the study of a number of interesting open questions concerning the effect of (normal) vibration on the dynamics of thin liquid films, including moving contact lines (Huh \& Scriven 1971), coarsening dynamics (Bestchorn et al. 2003; Merkt et al. 2005) and modes of instability of multilayer films (Pototsky et al. 2004, 2005). In particular, the influence of vibration on various transverse instabilities of advancing and receding contact lines (see, for instance, Cazabat el al. 1990; Brzoska, BrochardWyart \& Rondelez 1992; Spaid \& Homsy 1996; Veretennikov, Indeikina \& Chang 1998; Bertozzi et al. 1998; Dicz, Kondic \& Bcrtozzi 2001; Thicle \& Knobloch 2003) is of particular interest, both from a fundamental point of view and for practical applications. We also anticipate applications of the theory to the problem of levelling of a rough liquid surface (Schwartz et al. 1996), where we expect that an initially rippled stable thin liquid layer will level faster in the presence of vibration because the effective increase in surface tension 'hardens' the surface. Finally, we also expect that instabilities due to localized or heterogeneous heating (Kabov 1998; Skotheim, 'Thicle \& Scheid 2003; Kalliadasis, Kiyashko \& Demekhin 2003) may be controlled through the use of vibration, reducing for instance the probability of dry spots in heal exchangers.

This research was supported in part by $\mathrm{DGES}$, NASA and EU under Grants MI'M2004-03808, NNC04GA47G and MR'IN-C'I'-2004-005728.

\section{Appendix. Effect of thermal expansion}

In the derivation of the evolution equation we have neglected buoyancy effects due to density changes in the body of the film. 'To justify this assumption we permit the density to depend on temperature according to $\rho=\rho_{0}\left[1-\beta\left(T^{*}-T_{0}^{*}\right)\right]$, and include the resulting density changes whenever they couple to (frequency-modulated) gravity. 'This assumption constitutes the Boussinesq approximation and is amply satisfied in thin films. With the resulting additional term the vertical momentum equation (2.3) is replaced by

$$
\partial_{1}\left(w+\boldsymbol{u} \cdot \nabla(\omega)+w \partial_{z} \omega\right)=-\partial_{z} p+\nabla^{2} u+\partial_{z z}^{2} w-\varepsilon\left(\mathscr{G}+a(\omega)^{2} \cos \omega t\right) \theta .
$$

where

$$
\varepsilon=\beta\left(T_{0}^{*}-T_{1}^{*}\right) \ll 1
$$

is a measure of thermal expansion, and is related to the Rayleigh number, $R a=$ $g \beta\left(T_{0}^{*}-T_{1}^{*}\right) d^{* 3} /\left(k_{t h} \nu\right) \equiv \varepsilon \mathscr{C} P$. Proceeding as in $\$ 3$, we replace equations $(3.8 c)$ and 
$(3.12 a)$ by

$$
\partial_{z} p_{o}=-\frac{\varepsilon}{2} O_{s}, \quad \partial_{z} p_{s}=-\varepsilon\left[\varphi O_{s}+\frac{V i}{2}\left(O_{0}+\mathrm{ccc}\right)\right] \quad \text { in } 0<z<h_{s},
$$

and the boundary condition $(3.14 b)$ by

$$
p_{s}-\frac{V i}{2}\left[\left(\varepsilon \theta_{s}+1\right) h_{o}+\varepsilon\left(h_{s}-1\right) \theta_{o}+\text { c.c. }\right]-\mathscr{G}\left(h_{s}-1\right)+\tilde{\mathscr{S}} \widetilde{\nabla}^{2} h_{s}=0 \text { at } z=h_{s} .
$$

Since $\varepsilon$ is small but $\mathscr{G}$ cannot be too large (in order to avoid stabilization by gravity waves (Davis 1987) the new terms introduced by thermal expansion contribute only small corrections to the results obtained in $\$ 3$.

\section{REFERENCES}

BínsRd, H. 1900 Les tourbillons cellulaires dans une nappe liquide. Rev. Gén. Sci. Pures Appl. 11, $1261-1271$.

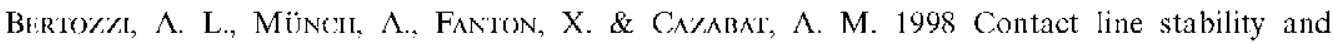
"undercompressive shocks" in driven thin film flow. Phys. Rev. Lett. 81, 51695173.

Bestehorn, M., Pototsky, A. \& Thiele, U. 2003 3D large scale Marangoni convection in liquid films. Eur. Phys. J. B 33, 457467.

BLOcK, M. J. 1956 Surface tension as the cause of Bénard cells and surface deformation in a liquid film. Nature 178, 650651 .

Boos, W. \& THEss, A. 1999 Cascade of structures in long-wavelength Marangoni instability. Phys. Fluids 11, 1484-1494.

Bragard, J. \& Velarde, M. G. 1998 Bénard-Marangoni convection: Planforms and related theoretical predictions. J. Filuid Mech. 368, 165-194.

Brzoska, J. B., Brochard-Wyart, F. \& Rondelez, F. 1992 Exponential growth of lingcring instabilities of spreading films under horizontal thermal gradients. Europhys. Lett. 19, 97-102.

Cazabat, A. M., Heslot, F., Troian, S. M. \& Carles, P. 1990 lingering instability of thin spreading films driven by temperature gradients. Nature 346, 824-826.

Chang, H.-C. 1989 Onset of nonlincar waves on lalling film. Phys. Fluids A 1, 1314-1327.

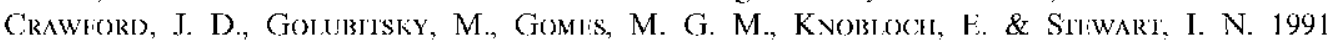
Boundary conditions as symmetry constraints. In Singularity Theory and its Applications. Part II, Lecture Notes in Mathematics, vol. 1463, pp. 63-79. Springer.

Cross, M. C. \& Hohenberg, P. C. 1993 Pattern formation out ol equilibrium. Rev. Mod. Phys. 65, $851-1112$.

DAvis, S. H. 1987 Thermocapillary instabilities. Annu. Rev. Fluid Mech. 19, 403435.

Diez, J. A., Kondic, L. \& Bertozzi, A. 2001 Global models for moving contact lines. Phys. Rev. H. $63,011208,113$.

Doedel, E. J., Champneys, A. R., Fairgrieve, T. F., Kuznetsov, Y. A., Sandstede, B. \& Wang, X. J. 1997 AUTO97: Continuation and Bifurcation Software for Ordinary Differential Equations. Montreal: Concordia University.

ENgel, A. \& Swift, J. B. 2000 Planform selection in two-layer Bénard-Marangoni convection. Phys. Rev. ト. 62, 6540 6553.

FARADAY, M. 1831 On a peculiar class of acoustical figures; and on certain forms assumed by groups of particles upon vibrating elastic surfaces. Phil. Trans. R. Soc. Lond. 121, 299-340.

Gavrilyuk, I., Lukovsky, I. \& Timokha, A. 2004 Two-dimensional variational vibrocquilibria and laraday's drops. Z. Angew. Math. Phys. 55, 1015-1033.

Gershli, G. Z., Kolesnikov, A. K., Legros, J. C. \& Myznikova, B. I. 1997 On the vibrational convective instability of a horizontal, binary-mixture layer with Soret effect. J. Filuid Mech. 330, 251-269.

Gershli, G. Z., Zhukhovitskil, E. M. \& Jurkov, I. S. 1970 Convective stability in presence of periodically varying parameter. J. Appl. Math. Mech. - USSR 34, 442-452.

Golovin, A. A., Nepomnyashchy, A. A. \& Pismen, L. M. 1994 Interaction between short-scale Marangoni convection and long-scale deformational instability. Phys. Fluids 6, 34-48. 
Gresho, P. M. \& SANi, R. L. 1970 Effects of gravity modulation on stability of a heated fluid layer. J. Fluid Mech. 40, 783-806.

Hui, C. \& Scrivin, L. F. 1971 Hydrodynamic model of steady movement of a solid/liquid/fluid contact line. J. Colloid Interface Sci. 35, 85-101.

Jlel, A., Burgess, J. M., MCCormick, W. D., Swift, J. B. \& Swinney, H. L. 2000 Surface tension-driven convection patterns in two liquid layers. Physica D 143, 169-186.

JCRISI, M. \& LösIR, W. 1990 Analysis of periodic non-rotational $u$ striations in $m_{0}$ single crystals due to non-steady thermocapillary convection. J. Cryst. Growth 102, 214222.

KaBov, O. A. 1998 Formation of regular structures in at lalling liquid film upon local heating. Themophys. Aeromech 5, 547-551.

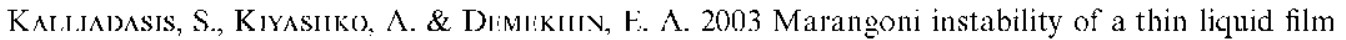
heated from below by a local heat source. I. Fluid Mech. 475, 377408.

Kataoka, D. E. \& Troian, S. M. 1997 A thcorctical study of instabilitics at the advancing front of thermally driven coating lilms. J. Colloid Interface Sci. 192, 350-362.

Koschmieder, E. 1993 Bénard Cells and Taylor Vortices. Cambridge University Press.

KuIImanN, H. C. 1999 Thermocapillary Contection in Models of Crystal Growth. Springer.

Kumar, K. \& Tuckerman, L. S. 1995 Parametric instability of the interlace between two lluids. J. Fluid Mech. 279, 49-68.

LANDAU, L. D. \& Lifshitz, E. M. 1987 Mechanics. Oxford University Press.

Laptulikix, V., Manelibo, F. J. \& Vi:cia, J. M. 2001 Control of Rayleigh-l'aylor instability by vertical vibration in large aspect ratio containcrs. Phys. Rev. E 64, 016318.

LIN, S. P. \& CheN, J. N. 1998 The mechanism of surface wave suppression in film flow down a vertical plane. Phys. Fluids 10, 1787-1792.

MANcibo, F. J. \& Vlici, J. M. 2002 Faraday instability threshold in large-aspect-ratio containers. J. Fluid Mech. 467, 307330.

Matar, O. K. \& Craster, R. V. 2001 Moddls lor Marangoni drying. Phys. Fluids 13, 1869-1883.

Merkt, D., Pototsky, A., Bestehorv, M. \& Thiele, U. 2005 Long-wave theory of bounded twolayer films with a free liquid-liquid interface: Short- and long-time evolution. Phys. Fluids 17, 064104.

Murray, B. T., Coriell, S. R. \& McFadden, G. B. 1991 The effect of gravity modulation on solutal convection during directional solidification. J. Gryst. Growth 110, 713-723.

NGUJYen, L. T. \& BAIAKoIAIAII, V. 2000 Modeling and experimental studies of wave evolution on frec lalling viscous lilms. Phys. Fluids 12, 2236-2256.

Nicolás, J. A., Rivas, D. \& VEGA, J. M. 1998 On the steady streaming flow due to high-frequency vibration in nearly inviscid liquid bridges. J. Filud Mech. 354, 147-174.

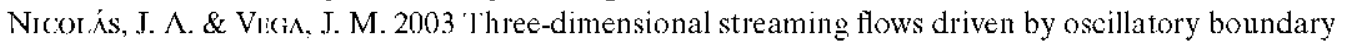
layers. Fluid Dyn. Res. 32, 119139.

OR, A. C. 1997 Finite-wavelength instability in a horizontal liquid layer on an oscillating planc. $J$. Fluid Mech. 335, 213-232.

OR, A. C. \& KIII.T, R. F. 2002 'T'he effects of thermal modulation upon the onset of MarangoniBénard convection. J. Fluid Mech. 456, 161182.

OR, A. C., Kelly, R. E., Cortelezzi, L. \& Speyer, J. L. 1999 Control ol long-wavelength MarangoniBinard convection. J. Fluid Mech. 387, 321-341.

Oron, A. 2000 Nonlinear dynamics of three-dimensional long-wave Marangoni instability in thin liquid films. Phys. Fluids 12, 16331645.

Oron, A., Davis, S. H. \& Barkoff, S. G. 1997 Long-scale cvolution of thin liquid films. Ret. Mod. Phys. 69, 931-980.

Pototsky, A., Bestehorn; M., Merkt, D. \& Thiele, U. 2004 Alternative pathways of dewetting for a thin liquid two-layer film. Phys. Rev. E 70, 025201(R).

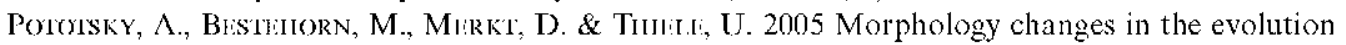
of licquid two-layer films. J. Chem. Phys. 122, 224711.

RAYLEIGH, LORD. 1916 On convective currents in a horizontal layer of fluid, when the higher temperature is on the under side. Phil. Mag. 6/32, 529-546.

Soimwari\%, L., CaIrnckoss, R. \& WiIIDNIR, D. 1996 nnomalous behavior during leveling of thin coating laycrs with surfactant. Phys. Fluids 8, 1693-1695.

Scriven, L. E. \& Sternling, C. V. 1960 Marangoni cllccts. Nature 187, 186-188. 
SKARDA, J. R. L. 2001 Instability of a gravity-modulated fluid layer with surface tension variation. J. Fluid Mech. 434, 243-271.

Skotheim, J. M., Thiele, U. \& Scheid, B. 2003 On the instability of a falling film due to localized heating. J. Fluid Mech. 475, 1-19.

SpaId, M. A. \& Homsy, G. M. 1996 Stability of Newtonian and viscoclastic dynamic contact lines. Phys. Fhids 8, 460-478.

Thess, A. \& Boos, W. 1999 A model for Marangoni drying. Phys. Fluids 11, 3852-3855.

Thiele, U. \& KNOBLOCH, E. 2003 Front and back instability of a liquid film on a slightly inclined platc. Phys. Fluids 15, 892-907.

Tiniti:, U. \& KNobiocit, F. 2004 'Thin liquid films on a slightly inclined heated plate. Physica 1) $190,213-248$.

Thitili, U., Veitaridi, M. G., Nitulitir, K., Bistlitorn, M. \& Pomiac; Y. 2001 Sliding drops in the difluse interlace model coupled to hydrodynamics. Phys. Rev. E 64, 061601, 1-12.

VanHook, S. J., Schim\%, M. F., M(Cormick, W. D., Swirt, J. B. \& Swinnily, H. L. 1995 Longwavelength instability in surlace-tension-driven Bénard convection. Phys. Ret. Lett. 75, 43974400 .

VanHook, S. J., Schim\%, M. F., Swili, J. B., MoCormick, W. D. \& Swinnily, H. L. 1997 Longwavelength surface-tension-driven Bénard convection: Experiment and theory. J. Flaid Mech. $345,45-78$.

Veretennikov, I., Indeikina, A. \& Chang, H.-C. 1998 Front dynamics and lingering of a driven contact linc. $J$. Fluid Mech. 373, 81-110.

Wheeler, A. A., McFadden, G. B., Murray, B. T. \& Coriell, S. R. 1991 Convective stability in the Rayleigh-Bénard and directional solidilication problems - high-frequency gravity modulation. Phys Fluids A 3, 2847-2858.

Wolf, G. H. 1970 Dynamic stabilization of interchange instability of a licuid-gas interlace. Phys. Rev. Lett. 24, 444-446.

Woots, D. R. \& Lin, S. P. 1995 Instability of a liquid film llow over a vibrating inclined plane. J. Fluid Mech. 294, 391-407. 